\title{
The Equivariant Bundle Subtraction Theorem and its applications
}

\author{
by \\ Masaharu Morimoto (Okayama) and \\ Krzysztof P awałows ki (Poznań)
}

Dedicated to the memory of Professor Katsuo Kawakubo

\begin{abstract}
In the theory of transformation groups, it is important to know what kind of isotropy subgroups of $G$ do occur at points of the space upon which the given group $G$ acts. In this article, for a finite group $G$, we prove the Equivariant Bundle Subtraction Theorem (Theorem 2.2) which allows us to construct smooth $G$-manifolds with prescribed isotropy subgroups around the $G$-fixed point sets. In Theorem 0.1 , we restate Oliver's result about manifolds $M$ and $G$-vector bundles over $M$ that occur, respectively, as the $G$-fixed point sets and their equivariant normal bundles for smooth $G$-actions on disks. In Theorems 0.2 and 0.3 , we prove the corresponding results for smooth $G$-actions on disks with prescribed isotropy subgroups around $M$. In Theorems 0.4 and 0.5 , for large classes of finite groups $G$, we explicitly describe manifolds $M$ that occur as the $G$-fixed point sets for such actions on disks. These actions are expected to be useful for answering the question of which manifolds occur as the $G$-fixed points sets for smooth $G$-actions on spheres.
\end{abstract}

0. Introduction. In the theory of transformation groups, it often happens that a solution of a particular problem depends on the family of the isotropy subgroups that we allow to occur at points in the space upon which a given group $G$ acts. In [O4], for a finite group $G$ not of prime power order, Oliver describes necessary and sufficient conditions under which a smooth manifold $M$ occurs as the $G$-fixed point set and a smooth $G$-vector $\nu$ over $M$ stably occurs as the equivariant normal bundle of $M$ in $D$ (resp., $E$ ) for a

1991 Mathematics Subject Classification: Primary 57S17, 57S25; Secondary 55M35, 55R91.

Key words and phrases: equivariant bundle subtraction, smooth action on disk, fixed point set, equivariant normal bundle, the family of large subgroups of a finite group.

Research of M. Morimoto partially supported by Grant-in-Aid for Scientific Research.

Research of K. Pawałowski partially supported by Polish Scientific Research Grant KBN 2 P03A 03115. 
smooth action of $G$ on a disk $D$ (resp., Euclidean space $E$ ). The conditions include two bundle conditions: a triviality condition and a condition dictated by the Smith Theory (see Theorem 0.1 for a more precise statement of Oliver's result in the case of actions on disks). The articles [EL], [P1], [P2] present some related partial results.

The main goal of the present article is to show that for actions on disks, Oliver's result remains true when we prescribe the family of isotropy subgroups that occur around the $G$-fixed point set $D^{G}=M$ (Theorems 0.2 and 0.3). The main new ingredient that allows us to prescribe the family of isotropy subgroups is the Equivariant Bundle Subtraction Theorem (Theorem 2.2). Roughly speaking, given a $G$-vector bundle $\xi$ over a finite $G$-CW complex $X$ and a real $G$-module $V$ with isotropy subgroups that we want to avoid around $D^{G}$ in the construction of action of $G$ on $D$, Theorem 2.2 describes circumstances under which we are able to subtract from $\xi$ the product $G$-vector bundle $\varepsilon_{X}^{V}$ over $X$ with fiber $V$, and as a result, to obtain a new $G$-vector bundle over $X$ with a prescribed family of isotropy subgroups. The subtraction is done by killing homotopy groups of the $\operatorname{space} \operatorname{Inj}_{H}(V, W)$ of $H$-linear injections of $V$ into $H$-modules $W$ for subgroups $H$ of $G$, where each $W$ contains the same (suitably chosen) stabilization summand.

For a prime $p$ dividing the order $|G|$ of $G$, denote by $\mathcal{P}_{p}(G)$ the family of all subgroups $P$ of $G$ of order $p^{a}$ with $a \geq 0$. We make use of the following two bundle conditions imposed on the Whitney sum $\tau_{M} \oplus \nu$, where $\tau_{M}$ is the tangent bundle of $M$. The conditions hold for $\tau_{M} \oplus \nu$ if and only if they hold for $\tau_{M} \oplus \nu \oplus \varepsilon_{M}^{W}$, where $W$ is a real $G$-module.

(B1) Product Bundle Condition. The bundle $\tau_{M} \oplus \nu$ is nonequivariantly a product bundle, perhaps after adding some product bundle, which amounts to saying that in the reduced $K O$-theory $\widetilde{K O}(M)$ of $M,\left[\tau_{M} \oplus \operatorname{Res}_{\{e\}}^{G}(\nu)\right]=0$ for the trivial subgroup $\{e\}$ of $G$.

(B2) Smith Theory Condition. For each prime $p|| G \mid$ and each $P \in$ $\mathcal{P}_{p}(G)$, in the reduced $P$-equivariant $K O$-theory $\widetilde{K O}_{P}(M)$ of $M$, the class $\left[\tau_{M} \oplus \operatorname{Res}_{P}^{G}(\nu)\right]$ is of finite order not divisible by $p$, which amounts to saying that $\left[\tau_{M} \oplus \operatorname{Res}_{P}^{G}(\nu)\right]=0$ in $\widetilde{K O}_{P}(M)_{(p)}$.

TheOrem 0.1 (Oliver [O4]). Let $G$ be a finite group not of prime power order. Let $M$ be a smooth manifold and let $\nu$ be a smooth $G$-vector bundle over $M$ with $\operatorname{dim} \nu^{G}=0$. Then the following two statements are equivalent:

(1) There exists a smooth action of $G$ on a disk $D$ such that $D^{G}=M$ and $\nu_{M \subset D} \cong \nu \oplus \varepsilon_{M}^{W}$ for a real G-module $W$ with $\operatorname{dim} W^{G}=0$.

(2) $M$ is compact, $\chi(M) \equiv 1\left(\bmod n_{G}\right)$, and $\tau_{M} \oplus \nu$ satisfies (B1) and (B2). 
The integer $n_{G}$ has been determined and computed by Oliver (cf. [O1], [O2], and [O3]) in the context of his work on the $G$-fixed point sets of finite contractible $G$-CW complexes for a finite group $G$ not of prime power order (see [O4, Theorem 0.3] for a summary of computation of $n_{G}$ ). The integer $n_{G}$ is defined as the unique nonnegative integer such that

$$
n_{G} \cdot \mathbb{Z}=\left\{\chi\left(X^{G}\right)-1 \mid X \text { is a finite contractible } G \text {-CW complex }\right\}
$$

by showing that the set is a subgroup of $\mathbb{Z}$ (cf. [O4, p. 597]). Theorem 0.1 allows us to answer the question of which manifolds $M$ occur as the $G$-fixed point sets for smooth actions of $G$ on disks (cf. [O4, Theorem 0.2]).

For a finite group $G$, we denote by $\mathcal{S}(G)$ the family of all subgroups of $G$. Each family of subgroups of $G$ that we consider is invariant under the action of $G$ on $\mathcal{S}(G)$ given via conjugation. Set

$$
\mathcal{P}(G)=\bigcup_{p} \mathcal{P}_{p}(G)
$$

Thus, $\mathcal{P}(G)$ is the family of subgroups $P$ of $G$ such that $P \subset G_{p}$ for a Sylow $p$-subgroup $G_{p}$ of $G$. For each prime $p$, define the Dress subgroup $G^{p}$ of $G$ of type $p$ as the smallest normal subgroup of $G$ such that $G / G^{p}$ is a $p$-group or the trivial group. Let $\mathcal{L}^{p}(G)$ be the family of subgroups $L$ of $G$ such that $L \supset G^{p}$. Define the family of large subgroups of $G$ by

$$
\mathcal{L}(G)=\bigcup_{p} \mathcal{L}^{p}(G)
$$

As in [LM], set $\mathcal{M}(G)=\mathcal{S}(G) \backslash \mathcal{L}(G)$ (see [LM, Proposition 1.2 and Theorem 1.3] for more information). Clearly, $\mathcal{P}(G) \subset \mathcal{M}(G)$ if and only if $\mathcal{P}(G) \cap \mathcal{L}(G)=\emptyset$, and the latter condition means that no large subgroup of $G$ has prime power order.

Following [LM], a finite group $G$ is called an Oliver group if $G$ is not of prime power order and $n_{G}=1$, which (by [O1]) amounts to saying that $G$ has no normal series of the form $P \unlhd H \unlhd G$, where $P$ nad $G / H$ are of prime power order and $H / P$ is cyclic. For a finite Oliver group $G$, the families $\mathcal{P}(G)$ and $\mathcal{L}(G)$ are disjoint.

In this article, we adopt the following notion. A finite group $G$ is called a Dress-Oliver group if each Dress subgroup of $G$ is an Oliver group. In particular, no Dress (and thus no large) subgroup of $G$ has prime power order; that is, $\mathcal{P}(G)$ and $\mathcal{L}(G)$ are disjoint.

If $G$ is a finite Dress-Oliver group, then each large subgroup of $G$ is an Oliver group because for any two subgroups $H \subset K$ of $G, n_{H}=1$ implies $n_{K}=1$. Hence, any finite Dress-Oliver group is an Oliver group.

Let $G$ be a finite nonsolvable group. Then $n_{G}=1$ (cf. [O2]). Let $G^{\text {sol }}$ be the smallest normal subgroup of $G$ such that $G / G^{\text {sol }}$ is solvable. Since $G^{\text {sol }}$ is a nontrivial perfect group, $G^{\text {sol }} \subset G^{p}$ for any Dress subgroup $G^{p}$ of 
$G$. Thus, $G^{p}$ is nonsolvable and $n_{G^{p}}=1$. Therefore, any finite nonsolvable group $G$ is a Dress-Oliver group.

A finite group $G$ is nilpotent if and only if $G$ is the product of its Sylow subgroups (equivalently, all Sylow subgroups of $G$ are normal subgroups of $G$ ). It follows that a finite nilpotent group $G$ is an Oliver group (resp., Dress-Oliver group) if and only if $G$ has three (resp., four) or more noncyclic Sylow subgroups.

For a $G$-space $X$, we denote by $\mathcal{F}_{\text {iso }}(G ; X)$ the family of the isotropy subgroups $G_{x}$ that occur at points $x \in X$. For a $G$-invariant subspace $A$ of $X$, we say that the action of $G$ on $X$ is without large isotropy subgroups around $A$ if there exists a $G$-invariant open neighborhood $U$ of $A$ in $X$ such that $\mathcal{F}_{\text {iso }}(G ; U \backslash A) \subset \mathcal{M}(G)$.

In this article, a real $G$-module $V$ is called $\mathcal{L}(G)$-free if $\operatorname{dim} V^{L}=0$ for each $L \in \mathcal{L}(G)$. Accordingly, a $G$-vector bundle $\nu$ over a manifold $M$ (with the trivial action of $G$ ) is called $\mathcal{L}(G)$-free if $\operatorname{dim} \nu^{L}=0$ for each $L \in \mathcal{L}(G)$. For a smooth $G$-manifold $D$ with $D^{G}=M$, set $\nu=\nu_{M \subset D \text {. By }}$ the Equivariant Tubular Neighborhood Theorem, the action of $G$ on $D$ is without large isotropy subgroups around $M$ if and only if $\nu$ is $\mathcal{L}(G)$-free.

Theorem 0.2. Let $G$ be a finite group such that $\mathcal{P}(G) \cap \mathcal{L}(G)=\emptyset$. Let $M$ be a smooth manifold and let $\nu$ be a smooth $\mathcal{L}(G)$-free $G$-vector bundle over $M$. Assume $n_{G}>1$ and $M$ has a connected component of dimension $>1$ (resp., $\left.n_{G}=0\right)$. Then the following two statements are equivalent:

(1) There exists a smooth action of $G$ on a disk $D$ such that $D^{G}=M$ and $\nu_{M \subset D} \cong \nu \oplus \varepsilon_{M}^{W}$ for a real $G$-module $W$. Moreover, the action of $G$ on $D$ is without large isotropy subgroups around $M$ (resp., in $D \backslash M)$.

(2) $M$ is compact, $\chi(M) \equiv 1\left(\bmod n_{G}\right)$, and $\tau_{M} \oplus \nu$ satisfies (B1) and (B2).

Theorem 0.3. Let $G$ be a finite Oliver group (resp., a finite DressOliver group). Let $M$ be a smooth manifold and let $\nu$ be a smooth $\mathcal{L}(G)$-free $G$-vector bundle over $M$. Then the following two statements are equivalent:

(1) There exists a smooth action of $G$ on a disk $D$ such that $D^{G}=M$ and $\nu_{M \subset D} \cong \nu \oplus \varepsilon_{M}^{W}$ for a real $G$-module $W$. Moreover, the action of $G$ on $D$ is without large isotropy subgroups around $M$ (resp., in $D \backslash M)$.

(2) $M$ is compact and $\tau_{M} \oplus \nu$ satisfies (B1) and (B2).

In Theorems 0.2 and 0.3 , (1) implies (2) by Theorem 0.1 , and (2) implies (1) by our arguments in Section 3. Our construction of action (described in Theorem 3.2) yields a stabilization summand $\varepsilon_{M}^{W}$ due to the equivariant bundle extension procedure which we apply here. The procedure goes back to [O4, Theorem 2.4]. In the present article, we use that procedure under circumstances described in the Equivariant Bundle Extension Theorem (The- 
orem 2.1). The Equivariant Bundle Subtraction Theorem (Theorem 2.2) allows us to control the isotropy subgroups in the $G$-module $W$ to the effect that

$$
\mathcal{F}_{\text {iso }}(G ; W \backslash\{0\})=\mathcal{M}(G) .
$$

Using the Equivariant Thickening Theorem (Theorem 3.1), we construct a smooth action of $G$ on a disk $D$ without large isotropy subgroups around $D^{G}$ (Theorem 3.2).

Let $\mathcal{C}_{\mathcal{N}}$ be the class of finite groups $G$ with a normal subgroup $N$ such that $G / N \cong \mathbb{Z}_{n}$, where $\mathbb{Z}_{n}$ is the cyclic group of order $n=p q r$ for some three distinct primes $p, q$, r. Clearly, $\mathcal{P}(G) \cap \mathcal{L}(G)=\emptyset$ for each $G \in \mathcal{C}_{\mathcal{N}}$. The class $\mathcal{C}_{\mathcal{N}}$ contains all finite nilpotent groups $G$ whose order is divisible by three or more distinct primes. For example, $\mathcal{C}_{\mathcal{N}}$ contains the abelian groups $G=\mathbb{Z}_{p q r}$ and $\mathbb{Z}_{p q r} \times \mathbb{Z}_{p}$ both with $n_{G}=0, G=\mathbb{Z}_{p q r} \times \mathbb{Z}_{p q r}$ with $n_{G}=1$, and $G=\mathbb{Z}_{p q r} \times \mathbb{Z}_{p q}$ with $n_{G}=p q$ for three distinct primes $p, q, r$.

Let $\mathcal{D}_{\mathcal{N}}$ be the class of finite groups $G$ with a normal subgroup $N$ such that $G / N$ is a perfect group with a subquotient isomorphic to the dihedral group of order $2 p q$ for two relatively prime integers $p, q \geq 2$. Clearly, such a group $G$ is a nonsolvable group, and thus $G$ is a Dress-Oliver group. In particular, $\mathcal{P}(G) \cap \mathcal{L}(G)=\emptyset$.

Recall a smooth manifold $M$ is called stably complex if the tangent bundle $\tau_{M}$ admits a complex structure, perhaps after adding a product bundle, which amounts to saying that $M$ has a smooth embedding into some Euclidean space, such that the normal bundle of the embedding admits a complex structure. In particular, the manifold $M$ is orientable and the connected components of $M$ all have the same parity.

TheOREM 0.4. Let $G$ be a finite group in $\mathcal{C}_{\mathcal{N}}$ such that $G$ has a normal Sylow 2-subgroup. Let $M$ be a smooth manifold. Assume $n_{G}>1$ and $M$ has a connected component of dimension $>1$ (resp., $\left.n_{G}=0\right)$. Then the following three statements are equivalent:

(1) There exists a smooth action of $G$ on a disk $D$ such that $D^{G}=M$.

(2) $M$ is compact, $\chi(M) \equiv 1\left(\bmod n_{G}\right)$, and $M$ is stably complex.

(3) There exists a smooth action of $G$ on a disk $D$ such that $D^{G}=M$ and the action of $G$ on $D$ is without large isotropy subgroups around $M$ (resp., in $D \backslash M$ ).

TheOREM 0.5. Let $G$ be a finite group in $\mathcal{C}_{\mathcal{N}}$ such that $G$ has a normal Sylow 2-subgroup and $n_{G}=1$ (resp., let $G$ be a finite group in $\mathcal{D}_{\mathcal{N}}$ ). Let $M$ be a smooth manifold. Then the following three statements are equivalent:

(1) There exists a smooth action of $G$ on a disk $D$ such that $D^{G}=M$.

(2) $M$ is compact and stably complex (resp., $M$ is compact). 
(3) There exists a smooth action of $G$ on a disk $D$ such that $D^{G}=M$ and the action of $G$ on $D$ is without large isotropy subgroups around $M$ (resp., in $D \backslash M$ ).

In Theorems 0.4 and $0.5,(1)$ and (2) are equivalent by making use of Theorem 0.1. This is already pointed out in [O4] for larger classes of finite groups $G$. By our arguments in Section 3, (2) implies (3). Clearly, (3) implies (1).

The main motivation for the work presented in this article is as follows. By applying and developing the equivariant surgery from [BM] and [M1][M3], we expect to convert smooth actions of $G$ on disks (constructed in Theorems $0.2-0.5$ ) into smooth actions of $G$ on spheres with prescribed $G$ fixed point sets (cf. [LMP], [LM], and [P3]). In fact, in a subsequent article for some classes of finite groups $G$, we convert smooth actions of $G$ on disks $D$ (without large isotropy subgroups around $D^{G}$ ) into smooth actions of $G$ on spheres $S$ such that $S^{G}=D^{G}$. As a result, we answer the question of which manifolds $M$ occur as the $G$-fixed point sets for smooth actions of $G$ on spheres.

We refer to $[\mathrm{Br}],[\mathrm{tD}]$, and $[\mathrm{K}]$ for background material on transformation groups that we use in this article. In a number of arguments, we make use of the fact that for a subgroup $H$ of $G$, the $H$-fixed point set $X^{H}$ is the sum of

$$
X^{=H}:=\left\{x \in X \mid G_{x}=H\right\} \text { and } X^{>H}:=\left\{x \in X \mid G_{x}>H\right\},
$$

where $G_{x}>H$ means that $G_{x} \supset H$ and $G_{x} \neq H$.

1. $\mathcal{L}(G)$-free $G$-vector bundles. Let $G$ be a finite group. By a real (resp., complex) $G$-module $V$ we mean a finite-dimensional real (resp., complex) vector space $V$ with an orthogonal (resp. unitary) action of $G$. We write $D(V)$ and $S(V)$, respectively, for the unit invariant disk and sphere in $V$. For a $G$-submodule $W$ of $V, V-W$ denotes the $G$-orthogonal complement of $W$ in $V$.

Using the canonical embedding $\mathbb{R}\left[G / G^{p}\right] \rightarrow \mathbb{R}[G]$ of the real regular $G / G^{p}$-module $\mathbb{R}\left[G / G^{p}\right]$ into the real regular $G$-module $\mathbb{R}[G]$ for each Dress subgroup $G^{p}$ of $G$, the article [LM] defines and makes a crucial use of the following real $G$-module:

$$
V(G)=(\mathbb{R}[G]-\mathbb{R})-\bigoplus_{G^{p}}\left(\mathbb{R}\left[G / G^{p}\right]-\mathbb{R}\right),
$$

where the summand $\mathbb{R}$ subtracted from $\mathbb{R}[G]$, as well as from $\mathbb{R}\left[G / G^{p}\right]$, has the trivial action of $G$. By [LM, Theorem 2.3], $\mathcal{F}_{\text {iso }}(G, V(G) \backslash\{0\})=\mathcal{M}(G)$. In particular, $V(G)$ is $\mathcal{L}(G)$-free, and so is $l V(G)$ for any integer $l \geq 1$. When $l$ is even, $l V(G)$ is the realification of the complex $G$-module $(l / 2) U(G)$, 
where

$$
U(G)=(\mathbb{C}[G]-\mathbb{C})-\bigoplus_{G^{p}}\left(\mathbb{C}\left[G / G^{p}\right]-\mathbb{C}\right) .
$$

We refer to $U(G)$ and $V(G)$, respectively, as to the complex and real regular $\mathcal{L}(G)$-free $G$-modules. The $G$-modules $U(G)$ and $V(G)$ are faithful.

Oliver's result recalled in Theorem 0.1 allows us to answer the question of which compact smooth manifolds $M$ with $\chi(M) \equiv 1\left(\bmod n_{G}\right)$ occur as the $G$-fixed point sets for smooth actions of $G$ on disks. The answer is just a restatement of Theorem 0.1, obtained in terms of the existence of a smooth $G$-vector bundle $\nu$ over $M$, and it reads as follows.

THEOREM 1.1 (Oliver [O4]). Let $G$ be a finite group not of prime power order. Let $M$ be a compact smooth manifold with $\chi(M) \equiv 1\left(\bmod n_{G}\right)$. Then the following two statements are equivalent:

(1) There exists a smooth action of $G$ on a disk $D$ such that $D^{G}=M$.

(2) There exists a smooth $G$-vector bundle $\nu$ over $M$ with $\operatorname{dim} \nu^{G}=0$ such that $\tau_{M} \oplus \nu$ satisfies the bundle conditions (B1) and (B2).

Similarly, in terms of the existence of a smooth $\mathcal{L}(G)$-free $G$-vector bundle $\nu$ over $M$, we can answer the question of which compact smooth manifolds $M$ with $\chi(M) \equiv 1\left(\bmod n_{G}\right)$ occur as the $G$-fixed point sets for smooth actions of $G$ on disks without large isotropy subgroups around $M$. The related restatements of Theorems 0.2 and 0.3 read as follows.

TheOREM 1.2. Let $G$ be a finite group such that $\mathcal{P}(G) \cap \mathcal{L}(G)=\emptyset$. Let $M$ be a compact smooth manifold with $\chi(M) \equiv 1\left(\bmod n_{G}\right)$. Assume $n_{G}>1$ and $M$ has a connected component of dimension $>1\left(\right.$ resp., $\left.n_{G}=0\right)$. Then the following two statements are equivalent:

(1) There exists a smooth action of $G$ on a disk $D$ such that $D^{G}=$ $M$ and the action is without large isotropy subgroups around $M$ (resp., in $D \backslash M)$.

(2) There exists a smooth $\mathcal{L}(G)$-free $G$-vector bundle $\nu$ over $M$ such that $\tau_{M} \oplus \nu$ satisfies the bundle conditions (B1) and (B2).

TheORem 1.3. Let $G$ be a finite Oliver group (resp., Dress-Oliver group). Let $M$ be a compact smooth manifold. Then the following two statements are equivalent:

(1) There exists a smooth action of $G$ on a disk $D$ such that $D^{G}=$ $M$ and the action is without large isotropy subgroups around $M$ (resp., in $D \backslash M)$.

(2) There exists a smooth $\mathcal{L}(G)$-free $G$-vector bundle $\nu$ over $M$ such that $\tau_{M} \oplus \nu$ satisfies the bundle conditions (B1) and (B2). 
Theorems 1.2 and 1.3 call for constructions of smooth $\mathcal{L}(G)$-free $G$-vector bundles $\nu$ over $M$ such that $\tau_{M} \oplus \nu$ satisfies the bundle conditions (B1) and (B2).

LEMMA 1.4. Let $G$ be a finite group and let $(V, W)$ be a pair of real (resp., complex) $G$-modules $V$ and $W$ such that the following two conditions hold:

(1) $\operatorname{Res}_{P}^{G}(V) \cong \operatorname{Res}_{P}^{G}(W)$ for each $P \in \mathcal{P}(G)$.

(2) $\operatorname{dim} V^{L}=1$ and $\operatorname{dim} W^{L}=0$ for each $L \in \mathcal{L}(G)$.

Let $M$ be a compact smooth manifold (resp., compact stably complex smooth manifold). Then there exists a smooth $\mathcal{L}(G)$-free $G$-vector bundle $\nu$ over $M$ such that $\tau_{M} \oplus \nu$ satisfies the bundle conditions (B1) and (B2).

Proof. Take a real (resp., complex) stable tangent bundle $\tau_{M}^{\text {st }}$ and a real (resp., complex) stable normal bundle $\nu_{M}^{\text {st }}$ of $M$. Similarly to [O4, the proof of Lemma 3.2(a)], consider the real (resp., complex) $G$-vector bundle over $M$ :

$$
\eta=\left(\tau_{M}^{\mathrm{st}} \otimes \varepsilon_{M}^{V}\right) \oplus\left(\nu_{M}^{\mathrm{st}} \otimes \varepsilon_{M}^{W}\right) .
$$

Then, as real (resp., complex) $G$-vector bundles, $\eta \cong \tau_{M}^{\text {st }} \oplus \nu$ with

$$
\nu=\left(\tau_{M}^{\mathrm{st}} \otimes \varepsilon_{M}^{V-V^{G}}\right) \oplus\left(\nu_{M}^{\mathrm{st}} \otimes \varepsilon_{M}^{W}\right) .
$$

It follows that $\nu$ is $\mathcal{L}(G)$-free and $\tau_{M}^{\text {st }} \oplus \nu$ satifies the bundle conditions (B1) and (B2). Hence, $\tau_{M} \oplus \nu$ also satisfies (B1) and (B2).

EXAMPLE 1.5. Let $p, q, r$ be three distinct primes. Set $n=p q r$ and write $\zeta_{n}$ for the primitive $n$th root of unity. Let $G=\mathbb{Z}_{n}=\left\langle a \mid a^{n}=1\right\rangle$. Let $V=V_{1} \oplus V_{2} \oplus V_{3}$ and $W=W_{1} \oplus W_{2} \oplus W_{3}$, where $V_{i}$ and $W_{i}(i=1,2,3)$ are the irreducible 1-dimensional complex $G$-modules with characters

$$
\begin{aligned}
\chi_{V}(a) & =\chi_{V_{1}}(a)+\chi_{V_{2}}(a)+\chi_{V_{3}}(a)=1+\zeta_{n}+\zeta_{n}, \\
\chi_{W}(a) & =\chi_{W_{1}}(a)+\chi_{W_{2}}(a)+\chi_{W_{3}}(a)=\zeta_{n}^{x}+\zeta_{n}^{y}+\zeta_{n}^{z},
\end{aligned}
$$

and the integers $x, y$, and $z$ are chosen so that the following holds:

$$
\begin{array}{llll}
x \equiv 0(\bmod p), & x \equiv 1(\bmod q), & & x \equiv 1(\bmod r), \\
y \equiv 1(\bmod p), & y \equiv 0(\bmod q), & y \equiv 1(\bmod r), \\
z \equiv 1(\bmod p), & z \equiv 1(\bmod q), & & z \equiv 0(\bmod r) .
\end{array}
$$

Then it follows that $\operatorname{Res}_{P}^{G}(V) \cong \operatorname{Res}_{P}^{G}(W)$ for each $P \in \mathcal{P}(G)$. Moreover, $\operatorname{dim} V^{L}=1$ and $\operatorname{dim} W^{L}=0$ for each $L \in \mathcal{L}(G)$.

Proposition 1.6. Let $G$ be a finite group in $\mathcal{C}_{\mathcal{N}}$. Let $M$ be a compact stably complex smooth manifold. Then there exists a smooth $\mathcal{L}(G)$-free $G$ vector bundle $\nu$ over $M$ such that $\tau_{M} \oplus \nu$ satisfies the bundle conditions (B1) and (B2). 
Pr o of. Since $G \in \mathcal{C}_{\mathcal{N}}$, there exists a normal subgroup $H$ of $G$ such that $G / H \cong \mathbb{Z}_{n}$, where $n=p q r$ for some three distict primes $p, q, r$. Take the pair $(V, W)$ of complex $\mathbb{Z}_{n}$-modules $V$ and $W$ constructed in Example 1.5. Via the epimorphism $G \rightarrow G / H \cong \mathbb{Z}_{n}$, consider $V$ and $W$ as complex $G$-modules. Then it follows that $\operatorname{Res}_{P}^{G}(V) \cong \operatorname{Res}_{P}^{G}(W)$ for each $P \in \mathcal{P}(G)$, and also one gets $\operatorname{dim} V^{L}=1$ and $\operatorname{dim} W^{L}=0$ for each $L \in \mathcal{L}(G)$. Now, Lemma 1.4 (the complex case) completes the proof.

ExAmple 1.7. Let $G=\mathbb{D}_{p q}$, the dihedral group of order $2 p q$ for two relatively prime integers $p, q \geq 2$. Then there exists a pair $(V, W)$ of real $G$-modules $V$ and $W$ such that $\operatorname{Res}_{P}^{G}(V) \cong \operatorname{Res}_{P}^{G}(W)$ for each $P \in \mathcal{P}(G)$, and such that $\operatorname{dim} V^{G}=1$ and $\operatorname{dim} W^{G}=0$. More generally, if $G$ is a finite group with a subgroup $H$ such that $H / N \cong \mathbb{D}_{p q}$ for a normal subgroup $N$ of $H$, then $G$ has a similar pair of real $G$-modules. In fact, first take the appropriate $\mathbb{D}_{p q}$-modules $V$ and $W$, and (via the epimorphism $H \rightarrow$ $\left.H / N \cong \mathbb{D}_{p q}\right)$ consider $V$ and $W$ as $H$-modules. Then take the induced $G$-modules $\operatorname{Ind}_{H}^{G}(V)$ and $\operatorname{Ind}_{H}^{G}(W)$ to obtain the required pair of real $G$ modules (cf. [O4, the proof of Lemma 3.1(b)].

Proposition 1.8. Let $G$ be a finite group in $\mathcal{D}_{\mathcal{N}}$. Let $M$ be a compact smooth manifold. Then there exists a smooth $\mathcal{L}(G)$-free $G$-vector bundle $\nu$ over $M$ such that $\tau_{M} \oplus \nu$ satisfies the bundle conditions (B1) and (B2).

Proof. Since $G \in \mathcal{D}_{\mathcal{N}}$, there exists an epimorphism $G \rightarrow H$ onto a perfect group $H$ which has a subquotient isomorphic to the dihedral group of order $2 p q$ for two relatively prime integers $p, q \geq 2$. By Example 1.7, there exists a pair $(V, W)$ of real $H$-modules $V$ and $W$ such that $\operatorname{Res}_{P}^{H}(V) \cong$ $\operatorname{Res}_{P}^{H}(W)$ for each $P \in \mathcal{P}(H), \operatorname{dim} V^{H}=1$ and $\operatorname{dim} W^{H}=0$. Via the epimorphism $G \rightarrow H$, consider $V$ and $W$ as real $G$-modules. Then it follows that $\operatorname{Res}_{P}^{G}(V) \cong \operatorname{Res}_{P}^{G}(W)$ for each $P \in \mathcal{P}(G)$. Since $H$ is perfect, $H^{p}=H$ for each Dress subgroup $H^{p}$ of $H$. Hence, $\mathcal{L}(H)=\{H\}$ and the epimorphism $G \rightarrow H$ maps each $L \in \mathcal{L}(G)$ onto $H$. Thus, $\operatorname{dim} V^{L}=1$ and $\operatorname{dim} W^{L}=0$ for each $L \in \mathcal{L}(G)$. Now, Lemma 1.4 (the real case) completes the proof.

In the proof of Proposition 1.6 (resp., 1.8), the $G$-modules $V$ and $W$ are not faithful when $H \neq G$. However, by adding to $V$ and $W$ the complex (resp., real) regular $\mathcal{L}(G)$-free $G$-module $U(G)$ (resp., $V(G)$ ), we obtain a similar pair of complex (resp., real) faithful $G$-modules. As already noted, according to [LM, Theorem 2.3],

$$
\mathcal{F}_{\text {iso }}(G ; S(V(G)))=\mathcal{F}_{\text {iso }}(G ; V(G) \backslash\{0\})=\mathcal{M}(G) .
$$

For each $H \in \mathcal{M}(G)$, we estimate $\operatorname{dim} V(G)^{H}$ in terms of the index $|G: H|$ of $H$ in $G$. 
Proposition 1.9. Let $G$ be a finite group not of prime power order, and let $H \in \mathcal{M}(G)$. If $|G: H|=p_{1}^{a_{1}} \ldots p_{n}^{a_{n}}$ for distinct primes $p_{1}, \ldots, p_{n}$ with $n \geq 2$ and $a_{1}, \ldots, a_{n} \geq 1$, then

$$
\operatorname{dim} V(G)^{H} \geq\left(p_{1}^{a_{1}}-1\right) \ldots\left(p_{n}^{a_{n}}-1\right) .
$$

If $|G: H|$ is a power of a prime $p$, then $\operatorname{dim} V(G)^{H} \geq p-1$ and for $p=2$, $\operatorname{dim} V(G)^{H}>2$. Hence, in each case of $|G: H|, \operatorname{dim} V(G)^{H} \geq 2$, and thus $\operatorname{dim} S(V(G))^{H} \geq 1$.

Proof. For any prime $p, H G^{p} \neq H$ because $H G^{p}=H$ implies $H \supset G^{p}$, which means that $H \notin \mathcal{M}(G)$, a contradiction. According to the definition of the $G$-module $V(G)$,

$$
\operatorname{dim} V(G)^{H}=(|G: H|-1)-\sum_{G^{p}}\left(\left|G: H G^{p}\right|-1\right)
$$

(cf. [LM, (2.2)]). Assume $|G: H|=p_{1}^{a_{1}} \ldots p_{n}^{a_{n}}$ for distinct primes $p_{1}, \ldots, p_{n}$ with $n \geq 2$ and $a_{i} \geq 1$ for $i=1, \ldots, n$. As noted above, $H G^{p_{i}} \neq H$, and thus $\left|G: H G^{p_{i}}\right|=p_{i}^{\overline{b_{i}}}$ for some $0 \leq b_{i}<a_{i}$. For $p \neq p_{i}, G=H G^{p}$, and thus $\left|G: H G^{p}\right|=1$. Hence, by using $(*)$,

$$
\begin{aligned}
\operatorname{dim} V(G)^{H} & =\left(p_{1}^{a_{1}} \ldots p_{n}^{a_{n}}-1\right)-\left[\left(p_{1}^{b_{1}}-1\right)+\ldots+\left(p_{n}^{b_{n}}-1\right)\right] \\
& \geq\left(p_{1}^{a_{1}} \ldots p_{n}^{a_{n}}-1\right)-\left[\left(p_{1}^{a_{1}}-1\right)+\ldots+\left(p_{n}^{a_{n}}-1\right)\right] \\
& \geq\left(p_{1}^{a_{1}}-1\right) \ldots\left(p_{n}^{a_{n}}-1\right) \geq 2 .
\end{aligned}
$$

Assume $|G: H|=p^{a}$ for a prime $p$ and $a \geq 1$. If $p^{a}=2$ (that is, $p=2$ and $a=1$ ), then $H$ is a normal subgroup of $G$, and thus $H \supset G^{2}$, which means that $H \notin \mathcal{M}(G)$, a contradiction. Therefore, $p^{a} \geq 3$ and for some $0 \leq b<a$, by using $(*)$ as above,

$$
\operatorname{dim} V(G)^{H}=\left(p^{a}-1\right)-\left(p^{b}-1\right)=\left(p^{a-b}-1\right) p^{b} \geq p-1 \geq 2
$$

for $p \geq 3$. For $p=2$, we have $a \geq 2$ (as $2^{a} \geq 3$ ), and thus $\operatorname{dim} V(G)^{H}=$ $\left(2^{a-b}-1\right) 2^{b} \geq 2$. Moreover, $\operatorname{dim} V(G)^{H} \geq 3$ for $b=0, \operatorname{dim} V(G)^{H} \geq 4$ for $b \geq 2$, and $\operatorname{dim} V(G)^{H} \geq 6$ for $a \geq 3$ and $b=1$. Now, assume $a=2$ and $b=1$ (with $p=2$ ). Note that the integer

$$
q:=\left|H G^{2}: H\right|=\left|G^{2}:\left(H \cap G^{2}\right)\right|
$$

divides 4 . Since $H G^{2} \neq H$, it follows that $q>1$. For $q=2, H \cap G^{2}$ is a normal subgroup of $G$ with 2-power index. This implies that $H \supset H \cap G^{2} \supset G^{2}$, and thus $H \notin \mathcal{M}(G)$, a contradiction. For $q=4$, we have $b=0$, which contradicts the assumption $b=1$. Thus, the case $a=2$ and $b=1$ (with $p=2)$ is impossible. Therefore, $\operatorname{dim} V(G)^{H}>2$ for $p=2$.

2. The Equivariant Bundle Subtraction Theorem. In order to construct $G$-vector bundles over finite contractible $G$-CW complexes, we make use of the equivariant bundle extension procedure described by Oliver 
[O4]. For a finite $G$-CW complex $Y$ with nonempty $G$-fixed point set $F$, take the wedge

$$
Y_{F}=(Y / F) \vee F
$$

of the quotient space $Y / F$ and $F$ with respect to the base point $F \in Y / F$ and a chosen base point $x_{0} \in F$. For a $G$-vector bundle $\eta$ over $F$, consider the fiber $V_{0}$ of $\eta$ over $x_{0}$ as a real $G$-module. We show that the following theorem is a special case of the result from [O4, Theorem 2.4].

Theorem 2.1 (The Equivariant Bundle Extension Theorem). Let $G$ be a finite group not of prime power order. Let $Y, F, x_{0}$, and $V_{0}$ be as above. Assume that $Y$ is contractible and $\left[\operatorname{Res}_{\{e\}}^{G}(\eta)\right]=0$ in $\widetilde{K O}(F)$, and $\left[\operatorname{Res}_{P}^{G}(\eta)\right]=0$ in $\widetilde{K O}_{P}(F)_{(p)}$ for each $P \in \mathcal{P}_{p}(G)$ and each prime $p|| G \mid$. Then there exist a finite contractible $G-C W$ complex $X$ and a $G$-vector bundle $\xi$ over $X$ such that the following three conditions hold:

(1) $X \supset Y_{F}$ as a $G$-invariant subcomplex.

(2) $\mathcal{F}_{\text {iso }}\left(G ; X \backslash Y_{F}\right) \subset \mathcal{P}(G)$, and thus $X^{G}=F$.

(3) $\left.\xi\right|_{F} \cong \eta \oplus \varepsilon_{F}^{V}$ and $\left.\xi\right|_{Y / F} \cong \varepsilon_{Y / F}^{V_{0} \oplus V}$ for a real $G$-module $V$.

If $Y^{P}$ is simply connected for some $P \in \mathcal{P}_{p}(G)$ and a prime $p|| G \mid$, then the fundamental group $\pi_{1}\left(X^{P}\right)$ is a finite abelian group of order prime to $p$.

Proof. Let $\bar{\eta}$ be the $G$-vector bundle over $Y_{F}$ that restricts to $\eta$ over $F$ and the product $G$-vector bundle over $Y / F$ with fiber $V_{0}$. For each $P \in \mathcal{P}(G)$, let $\xi_{P}$ be the product $P$-vector bundle over $Y$ with fiber $\operatorname{Res}_{P}^{G}\left(V_{0}\right)$. The constant map $Y / F \rightarrow Y$ into the base point $x_{0} \in F$ and the inclusion $F \rightarrow Y$ piece together to a $G$-map $\varphi: Y_{F} \rightarrow Y$. Since $\left[\operatorname{Res}_{\{e\}}^{G}(\eta)\right]=0$ in $\widetilde{K O}(F)$, and $\left[\operatorname{Res}_{P}^{G}(\eta)\right]=0$ in $\widetilde{K O}_{P}(F)_{(p)}$,

$$
\left[\varphi^{*}\left(\xi_{\{e\}}\right)\right]=\left[\operatorname{Res}_{\{e\}}^{G}(\bar{\eta})\right] \text { and }\left[\varphi^{*}\left(\xi_{P}\right)\right]=\left[\operatorname{Res}_{P}^{G}(\bar{\eta})\right]
$$

respectively, in $\widetilde{K O}_{\{e\}}\left(Y_{F}\right)$ and $\widetilde{K O}_{P}\left(Y_{F}\right)_{(p)}$ for each nontrivial $P \in \mathcal{P}_{p}(G)$ and $p|| G \mid$. Therefore, it follows from [O4, Theorem 2.4, finite case] that we can combine $\bar{\eta}$ and all $\xi_{P}$ to construct a finite contractible $G$-CW complex $X$ and a $G$-vector bundle $\xi$ over $X$ such that $X \supset Y_{F}$ as a $G$-invariant subcomplex,

$$
\mathcal{F}_{\text {iso }}\left(G ; X \backslash Y_{F}\right) \subset \mathcal{P}(G) \text { and }\left.\quad \xi\right|_{Y_{F}} \cong \bar{\eta} \oplus \varepsilon_{Y_{F}}^{V}
$$

for a real $G$-module $V$. The essential part of the proof of [O4, Theorem 2.4, finite case] follows from [O4, Proposition 2.3, finite case], whose proof consists of two steps. The first step is made up of equivariant cell attachment of isotropy type $(P)$ with nontrivial $P \in \mathcal{P}(G)$, and equivariant bundle extension arguments. The second step is made up of attachment of free cells and equivariant bundle extension arguments. In particular, one extends the 
$G$-map $\varphi$ to a $G$-map $\bar{\varphi}: X \rightarrow Y$ that is a (nonequivariant) homotopy equivalence. The main part of the proof of the first step follows by the arguments given in the proof of [O4, Lemma 2.2, finite case]. The arguments include the claim that for each $P \in \mathcal{P}_{p}(G)$ and $p|| G \mid$, the kernel

$$
\operatorname{Ker}\left[\pi_{1}\left(\bar{\varphi}^{P}\right): \pi_{1}\left(X^{P}\right) \rightarrow \pi_{1}\left(Y^{P}\right)\right]
$$

is a finite abelian group of order prime to $p$. Therefore, if $Y^{P}$ is simply connected, then $\pi_{1}\left(X^{P}\right)$ is a finite abelian group of order prime to $p$.

As follows from [O4, Theorem 2.4], the $G$-module $V$ in Theorem 2.1 can be taken to be $l \mathbb{R}[G]$ or $l(\mathbb{R}[G]-\mathbb{R})$ for a sufficiently large integer $l$. In particular, all (proper) subgroups of $G$ do occur as the isotropy subgroups in $S(V)$. Now, we present a procedure which allows us to choose the stabilization summand $V$ so that $S(V)$ is without large isotropy subgroups. More specifically,

$$
\mathcal{F}_{\text {iso }}(G ; S(V))=\mathcal{S}(G) \backslash \mathcal{L}(G)=\mathcal{M}(G) .
$$

Let $G$ be a finite group and let $H$ be a subgroup of $G$. Denote by $\operatorname{Irr}(H)$ the set of the isomorphism classes of real irreducible $H$-modules, and for a real $H$-module $W$, denote by $\operatorname{Irr}(H ; W)$ the set of the isomorphism classes of the real irreducible $H$-modules which occur as $H$-submodules of $W$. A real $G$-module $V$ is called $H$-complete if

$$
\operatorname{Irr}(H)=\operatorname{Irr}\left(H ; \operatorname{Res}_{H}^{G}(V)\right),
$$

which amounts to saying that $\operatorname{Res}_{H}^{G}(V)$ contains (up to $H$-isomorphism) each irreducible $H$-module with positive multiplicity. For a family $\mathcal{H}$ of subgroups of $G$, a real $G$-module $V$ is called $\mathcal{H}$-complete if $V$ is $H$-complete for each $H \in \mathcal{H}$.

Theorem 2.2 (The Equivariant Bundle Subtraction Theorem). Let $G$ be a finite group. Let $V$ be a real $G$-module and let $V^{\prime}$ be a real $\mathcal{H}$-complete $G$-module for a family $\mathcal{H}$ of subgroups of $G$. Let $(X, Y)$ be a pair of finite $G-C W$ complexes such that $X \supset Y$ as a $G$-invariant subcomplex, and $\mathcal{F}_{\text {iso }}(G ; X \backslash Y) \subset \mathcal{H}$. Let $\xi$ and $\eta$ be $G$-vector bundles over $X$ and $Y$, respectively, such that

$$
\left.\xi\right|_{Y} \cong \eta \oplus \varepsilon_{Y}^{V} \oplus \varepsilon_{Y}^{l V^{\prime}} \quad \text { for an integer } l \geq 1 .
$$

If $l$ is sufficiently large, then there exists a $G$-subbundle $\theta$ of $\xi$ such that $\theta \cong \varepsilon_{X}^{V}$ and $\left.(\xi-\theta)\right|_{Y} \cong \eta \oplus \varepsilon_{Y}^{l V^{\prime}}$, where $\xi-\theta$ is the $G$-orthogonal complement of $\theta$ in $\xi$.

Proof. Choose a filtration $Y=X_{0} \subset X_{1} \subset \ldots \subset X_{n}=X$ of $X$ such that $X_{j}$ is obtained from $X_{j-1}$ by attaching just one equivariant cell of type $(H) \subset \mathcal{H}$ for $j=1, \ldots, n$. It suffices to prove the result for $n=1$ because then, for any $n \geq 1$, the general case follows by applying the arguments successively to each pair $\left(X_{j}, X_{j-1}\right)$. 
So, assume that $X$ is obtained from $Y$ by attaching a $G$-cell of the form $G / H \times D^{k}$ with $k \geq 0$ and $(H) \subset \mathcal{H}$. In particular, $V^{\prime}$ is $H$-complete; that is, $\operatorname{Res}_{H}^{G}\left(V^{\prime}\right)$ contains each irreducible $H$-module with positive multiplicity. Since $\left.\xi\right|_{Y} \cong \eta \oplus \varepsilon_{Y}^{V} \oplus \varepsilon_{Y}^{l V^{\prime}}$, there exists a $G$-subbundle $\zeta$ of $\left.\xi\right|_{Y}$ isomorphic to $\varepsilon_{Y}^{V}$. We claim that if $l$ is sufficiently large, $\zeta$ extends to a $G$-subbundle $\theta$ of $\xi$ isomorphic to $\varepsilon_{X}^{V}$. Once the claim is proven, we subtract $\theta$ from $\xi$; that is, we take the $G$-orthogonal complement $\xi-\theta$ of $\theta$ in $\xi$. Then $\left.(\xi-\theta)\right|_{Y} \cong \eta \oplus \varepsilon_{Y}^{l V^{\prime}}$. Thus it suffices to prove the claim.

First, assume $k=0$. Then we have to deal with $G$-vector bundles over $G / H$, or equivalently, $H$-modules over the point $\{e H\}$. If $l$ is sufficiently large, $\operatorname{Res}_{H}^{G}\left(l V^{\prime}\right)$ contains $\operatorname{Res}_{H}^{G}(V)$ by assumption, and the claim follows.

Assume $k>0$. Set $D^{k}=\{e H\} \times D^{k}$ and $S^{k-1}=\{e H\} \times S^{k-1}$. Let $f: S^{k-1} \rightarrow Y$ and $h: D^{k} \rightarrow X$ be, respectively, the restrictions of an attaching map and a characteristic map of the equivariant cell $G / H \times D^{k}$ such that $f=\left.h\right|_{S^{k-1}}$. Let $W$ be the $H$-module determined on the fiber of $\xi$ over the origin $h(0)$ of the attached disk $h\left(D^{k}\right) \subset X$. Since $\zeta \cong \varepsilon_{Y}^{V}$ and $h^{*} \xi$ is a product $H$-vector bundle over $D^{k}$, there exist $H$-trivializations

$$
\tau: S^{k-1} \times V \rightarrow E\left(f^{*} \zeta\right) \text { and } \sigma: D^{k} \times W \rightarrow E\left(h^{*} \xi\right)
$$

of the induced $H$-vector bundles $f^{*} \zeta$ and $h^{*} \xi$. The trivializations $\tau$ and $\sigma$ differ over $S^{k-1}$ by a map

$$
d_{\tau, \sigma}: S^{k-1} \rightarrow \operatorname{Inj}_{H}(V, W)
$$

from $S^{k-1}$ into the space of $H$-linear injections of $V$ into $W$. Once $d_{\tau, \sigma}$ extends over $D^{k}, f^{*} \zeta$ extends to an $H$-subbundle of $h^{*} \xi$ isomorphic to the product $H$-vector bundle over $D^{k}$ with fiber $\operatorname{Res}_{H}^{G}(V)$, which amounts to saying that $\zeta$ extends to a $G$-subbundle $\theta$ of $\xi$ isomorphic to $\varepsilon_{X}^{V}$. Therefore, to complete the proof of the claim, it suffices to show that $d_{\tau, \sigma}$ does extend over $D^{k}$. To do this, let us analyse the space $\operatorname{Inj}_{H}(V, W)$. Let $\left\{W_{i}\right\}_{i \in \mathcal{I}}$ be a complete (finite) set of irreducible real $H$-modules. Then, as $H$-modules,

$$
\operatorname{Res}_{H}^{G}(V) \cong \bigoplus_{i \in \mathcal{I}} n_{i} W_{i} \quad \text { and } \quad \operatorname{Res}_{H}^{G}\left(V^{\prime}\right) \cong \bigoplus_{i \in \mathcal{I}} n_{i}^{\prime} W_{i}
$$

for some integers $n_{i}, n_{i}^{\prime} \geq 0$. Since $V^{\prime}$ is $H$-complete, $n_{i}^{\prime}>0$ for each $i \in \mathcal{I}$. Recall that $\left.\xi\right|_{Y} \cong \eta \oplus \varepsilon_{Y}^{V} \oplus \varepsilon_{Y}^{l V^{\prime}}$. Hence, $W \supset \operatorname{Res}_{H}^{G}\left(V \oplus l V^{\prime}\right)$ as an $H$ submodule, and thus

$$
W \cong \bigoplus_{i \in \mathcal{I}}\left(n_{i}+l_{i}\right) W_{i} \quad \text { with } l_{i}=l n_{i}^{\prime}+c_{i}
$$

for some integers $c_{i} \geq 0$. For each $i \in \mathcal{I}$, put $E_{i}=\operatorname{End}_{H}\left(W_{i}\right)$. Then $E_{i} \cong \mathbb{R}$, $\mathbb{C}$, or $\mathbb{H}$. Clearly,

$$
\varepsilon_{S^{k-1}}^{n_{i} W_{i}} \cong \varepsilon_{S^{k-1}}^{n_{i} E_{i}} \otimes_{E_{i}} \varepsilon_{S^{k-1}}^{W_{i}} \quad \text { and } \quad \varepsilon_{D^{k}}^{\left(n_{i}+l_{i}\right) W_{i}} \cong \varepsilon_{D^{k}}^{\left(n_{i}+l_{i}\right) E_{i}} \otimes_{E_{i}} \varepsilon_{D^{k}}^{W_{i}} .
$$


For each $i \in \mathcal{I}$, it follows from Schur's lemma that an $H$-linear injection $V \rightarrow W$ maps $n_{i} W_{i}$ into $\left(n_{i}+l_{i}\right) W_{i}$. Therefore,

$$
\operatorname{Inj}_{H}(V, W) \cong \prod_{i \in \mathcal{I}} \operatorname{Inj}_{H}\left(n_{i} W_{i},\left(n_{i}+l_{i}\right) W_{i}\right) .
$$

Let $O_{n}\left(E_{i}\right)$ denote the group of metric-preserving automorphisms of the $n$-fold $E_{i}^{n}$ of $E_{i}$ for a given integer $n \geq 1$; that is, $O_{n}(\mathbb{R})=O(n), O_{n}(\mathbb{C})=$ $U(n)$, and $O_{n}(\mathbb{H})=\operatorname{Sp}(n)$. For each $i \in \mathcal{I}$, choose an orthonormal $n_{i}$-frame in $E_{i}^{n_{i}+l_{i}}$ to obtain a splitting

$$
\operatorname{Inj}_{H}(V, W) \cong \prod_{i \in \mathcal{I}} O_{n_{i}+l_{i}}\left(E_{i}\right) / O_{l_{i}}\left(E_{i}\right) .
$$

Put $e_{i}=1,2$, or 4 when $E_{i} \cong \mathbb{R}, \mathbb{C}$, or $\mathbb{H}$, respectively. Choose $l$ large enough to ensure that $k-1 \leq\left(l_{i}+1\right) e_{i}-2$ for each $i \in \mathcal{I}$ (recall $l_{i}=l n_{i}^{\prime}+c_{i}$ ). It follows that $\pi_{k-1}\left(O_{n_{i}+l_{i}}\left(E_{i}\right) / O_{l_{i}}\left(E_{i}\right)\right)=0$ (cf. [H, Theorem 5.1, p. 95]). Thus, the homotopy class

$$
\omega:=\left[d_{\tau, \sigma}\right] \in \pi_{k-1}\left(\operatorname{Inj}_{H}(V, W)\right)
$$

vanishes; that is, $\omega=0$, which shows that $d_{\tau, \sigma}$ extends over $D^{k}$, proving the claim and hence the theorem.

Proposition 2.3. For a finite group $G$, the real regular $\mathcal{L}(G)$-free $G$ module $V(G)$ is $\mathcal{M}(G)$-complete, where $\mathcal{M}(G)=\mathcal{S}(G) \backslash \mathcal{L}(G)$.

Proof. Let $H \in \mathcal{M}(G)$. It suffices to show that $\operatorname{Res}_{H}^{G}(V(G)) \supseteq \mathbb{R}[H]$. Clearly, $H \subseteq H G^{p}$ for any prime $p$. If $H=H G^{p}$ for a prime $p$, then $H \supseteq G^{p}$; that is, $H \in \mathcal{L}(G)$, which gives a contradiction. Thus, for each prime $p, H \neq H G^{p}$. By the definition of $V(G)$,

$$
\operatorname{Res}_{H}^{G}(V(G))=\operatorname{Res}_{H}^{G}(\mathbb{R}[G]-\mathbb{R})-\bigoplus_{p|| G \mid} \operatorname{Res}_{H}^{G}\left(\mathbb{R}\left[G / G^{p}\right]-\mathbb{R}\right) .
$$

By Frobenius reciprocity, $\operatorname{Res}_{H}^{G}(\mathbb{R}[G])=|G / H| \mathbb{R}[H]$ and for each prime $p$,

$$
\operatorname{Res}_{H}^{G}\left(\mathbb{R}\left[G / G^{p}\right]\right)=\left|G / H G^{p}\right| \mathbb{R}\left[H /\left(H \cap G^{p}\right)\right] .
$$

Note that the group $H /\left(H \cap G^{p}\right)$ is a $p$-group, $\mathbb{R}[H] \supseteq \mathbb{R}\left[H /\left(H \cap G^{p}\right)\right]$, and

$$
\mathbb{R}[H]-\mathbb{R} \supseteq \bigoplus_{p|| G \mid}\left(\mathbb{R}\left[H /\left(H \cap G^{p}\right)\right]-\mathbb{R}\right) .
$$

Let $n$ denote the number of distinct primes dividing $|G|$. Then it follows that

$$
\begin{aligned}
\operatorname{Res}_{H}^{G}(V(G)) & =(|G / H| \mathbb{R}[H]+(n-1) \mathbb{R})-\bigoplus_{p \| G \mid}\left|G / H G^{p}\right| \mathbb{R}\left[H /\left(H \cap G^{p}\right)\right] \\
& \supseteq(|G / H|-m)(\mathbb{R}[H]-\mathbb{R}),
\end{aligned}
$$


where $m=\max \left\{\left|G / H G^{p}\right| \mid p\right.$ divides $\left.|G|\right\}$. Since $\operatorname{dim} V(G)^{H}>0$, we have $\operatorname{Res}_{H}^{G}(V(G)) \supseteq \mathbb{R}$, and since $H \neq H G^{p}$, it follows that $|G / H|-m>0$. Therefore, $\operatorname{Res}_{H}^{G}(V(G)) \supseteq \mathbb{R}[H]$.

Remark 2.4. Let $G$ be a finite group. Let $V$ be a real $G$-module and let $W$ be a $G$-submodule of $V$ such that $W \supset V^{L}$ for each $L \in \mathcal{L}(G)$; possibly, $W=V$. Then the $G$-orthogonal complement $V-W$ of $W$ in $V$ is $\mathcal{L}(G)$-free. By Proposition 2.3, $V(G)$ is $\mathcal{M}(G)$-complete. Hence, $(V-W) \oplus V(G)$ is both $\mathcal{M}(G)$-complete and $\mathcal{L}(G)$-free. In particular, if $V$ is $\mathcal{L}(G)$-free, then $V \oplus V(G)$ is both $\mathcal{M}(G)$-complete and $\mathcal{L}(G)$-free.

3. Constructions of smooth $G$-actions on disks. In order to construct smooth $G$-actions on disks, we employ the equivariant thickening procedure described in [P2] for any compact Lie group $G$. Roughly saying, given a finite $G$-CW complex $X$, a compact smooth $G$-manifold $M$ such that $X \supset M$, and a smooth $G$-vector bundle $\nu$ over $M$, the procedure allows us to build up $X$ into a compact smooth $G$-manifold $D$ of the $G$-homotopy type of $X$, such that $D \supset M$ as a $G$-submanifold with equivariant normal bundle $\nu_{M \subset D}$ stably isomorphic to $\nu$. This is done by making use of an appropriate $G$-vector bundle $\xi$ over $X$ which stably extends $\tau_{M} \oplus \nu$. In this article, we apply the equivariant thickening procedure for a finite group $G$, and we restate the results in the form convenient for our applications.

Theorem 3.1 (The Equivariant Thickening Theorem). Let $G$ be a finite group. Let $(M, \nu)$ be a pair consisting of a compact smooth $G$-manifold $M$ and a smooth $G$-vector bundle $\nu$ over $M$. Let $(X, \xi)$ be a pair consisting of a finite $G-C W$ complex $X$ and a $G$-vector bundle $\xi$ over $X$ such that the following three conditions hold:

(1) $X \supset M$ as a $G$-invariant subcomplex.

(2) $\mathcal{F}_{\text {iso }}(G ; X \backslash M) \subset \mathcal{F}_{\text {iso }}(G ; S(V))$ for a real $G$-module $V$ with $\operatorname{dim} V>0$.

(3) $\left.\xi\right|_{M} \oplus \varepsilon_{M}^{W} \cong \tau_{M} \oplus \nu \oplus \varepsilon_{M}^{W}$, where $W=l V$ for an integer $l \geq 1$.

If $l$ is sufficiently large, there exists a compact smooth $G$-manifold $D$ such that $D \supset M$ as a $G$-submanifold, $\nu_{M \subset D} \cong \nu \oplus \varepsilon_{M}^{W}$ as $G$-vector bundles, and

$$
\mathcal{F}_{\text {iso }}(G ; D \backslash M)=\mathcal{F}_{\text {iso }}(G ; S(\nu)) \cup \mathcal{F}_{\text {iso }}(G ; S(V))
$$

when $\mathcal{F}_{\text {iso }}(G ; S(V))$ is closed under taking subgroups. Moreover, $D \supset X$ as a $G$-invariant subcomplex, and there exists a strong $G$-deformation retraction $f: D \rightarrow X$ such that $\tau_{D} \cong f^{*}(\xi) \oplus \varepsilon_{D}^{W}$. In particular, $\left.\tau_{D}\right|_{X} \cong \xi \oplus \varepsilon_{X}^{W}$. If $X$ is (nonequivariantly) contractible, then $D$ can be chosen to be the disk of dimension $n=\operatorname{dim}\left(\tau_{M} \oplus \nu\right)+l \operatorname{dim} V \geq 6$.

Proof. Let $X_{0}$ be the sum of $M$ and all equivariant 0 -cells $G / H$ in $X \backslash M$. Extend $\tau_{M} \oplus \nu$ to a smooth $G$-vector bundle $\nu_{X_{0}}$ over $X_{0}$ by letting 
$\nu_{X_{0}}$ be $G \times_{H} F_{x}(\xi)$ over any equivariant 0 -cell $G / H$ in $X \backslash M$, where $F_{x}(\xi)$ is the fiber of $\xi$ over $x=e H \in G / H$, considered as an $H$-module. Recall $W=l V$ for an integer $l \geq 1$, and note that

$$
\left.\left(\xi \oplus \varepsilon_{X}^{W}\right)\right|_{X_{0}} \cong \tau_{X_{0}} \oplus \nu_{X_{0}} \oplus \varepsilon_{X_{0}}^{W} .
$$

Thus, the Normal Bundle Condition (NB) of [P2, p. 279] holds for $\xi \oplus \varepsilon_{X}^{W}$ and $\nu_{X_{0}} \oplus \varepsilon_{X_{0}}^{W}$. Since $\mathcal{F}_{\text {iso }}(G ; V)$ is closed under taking intersections, $V$ and $W$ have the same isotropy subgroups and the assumption $\mathcal{F}_{\text {iso }}(G ; X \backslash M) \subset$ $\mathcal{F}_{\text {iso }}(G ; S(V))$ implies that

$$
\mathcal{F}_{\text {iso }}\left(G ; X \backslash X_{0}\right) \subset \mathcal{F}_{\text {iso }}\left(G ; S\left(\nu_{X_{0}} \oplus \varepsilon_{X_{0}}^{W}\right)\right) .
$$

Thus, the Isotropy Subgroup Condition (IS) of [P2, p. 279] holds for $\nu_{X_{0}} \oplus$ $\varepsilon_{X_{0}}^{W}$. Moreover, the General Position Condition (GP) of [P2, p. 279] holds for $\nu_{X_{0}} \oplus \varepsilon_{X_{0}}^{W}$ when $l$ is sufficiently large. In fact, each isotropy subgroup $H$ that occurs in $X \backslash X_{0}$ occurs also in $S(W)$ and for each isotropy subgroup $H$ that occurs in $S(W)$, we can make both

$$
\operatorname{dim} W^{H} \text { and } \operatorname{dim} W^{H}-\operatorname{dim} W^{>H}
$$

as large as we wish by taking $l$ sufficiently large.

Choose a filtration $X_{0} \subset X_{1} \subset \ldots \subset X_{k}=X$ of $X$ such that $X_{j+1}$ is obtained from $X_{j}$ by attaching just one equivariant cell. Using (NB), (IS), and (GP), one constructs a sequence $D_{0} \subset D_{1} \subset \ldots \subset D_{k}$ of smooth $G$-manifolds $D_{j}$ such that $D_{j} \supset X_{j}$ for $j=0,1, \ldots, k$. One also constructs strong $G$-deformation retractions $f_{j}: D_{j} \rightarrow X_{j}$ such that $f_{j+1} \mid D_{j}=f_{j}$. The idea of the construction is to take $D_{0}$ and the bundle projection $f_{0}$ : $D_{0} \rightarrow X_{0}$, where $D_{0}$ is the total space $D\left(\nu_{X_{0}} \oplus \varepsilon_{X_{0}}^{W}\right)$ of the invariant unit disk bundle of $\nu_{X_{0}} \oplus \varepsilon_{X_{0}}^{W}$ over $X_{0}$. Then, in a way prescribed by $\xi \oplus \varepsilon_{X}^{W}$, one replaces each $m$-cell $G / H \times D^{m}$ in $X \backslash X_{0}$ by an equivariant $m$-handle $\left(G \times_{H} D(U)\right) \times D^{m}$ for an $H$-module $U$ (cf. [P2, Proposition 2.3]). By setting $D=D_{k}$ and $f=f_{k}$, one obtains a compact smooth $G$-manifold $D$ and a strong $G$-deformation retraction $f: D \rightarrow X$ such that $D \supset M$ as a $G$-submanifold, $\nu_{M \subset D} \cong \nu \oplus \varepsilon_{M}^{W}$, and $\tau_{D} \cong f^{*}(\xi) \oplus \varepsilon_{D}^{W}$ (cf. [P2, Theorem 2.4 and Remark 2.6]).

Note that $X_{0}$ is obtained from $M$ by adding equivariant 0 -cells $G / H$, and $D_{0}$ is obtained from $D\left(\nu \oplus \varepsilon_{M}^{W}\right)$ by replacing each $G / H$ in $X_{0} \backslash M$ by the equivariant 0-handle $G \times_{H} D\left(U_{x}\right)$ with $U_{x}=F_{x}(\xi) \oplus \operatorname{Res}_{H}^{G}(W)$ and $x=e H \in G / H$. In the inductive step of the construction, we replaced an equivariant $m$-cell of type $(H)$ by an equivariant $m$-handle of type $(H)$ for $m \geq 1$. For $m \geq 0$, the $m$-handle is a disk bundle over the $m$-cell. Thus, each isotropy subgroup in the handle of type $(H)$ is a subgroup of a conjugate of $H$. Let $\mathcal{H}$ be the family of the isotropy subgroups occurring in the added handles. If $\mathcal{F}_{\text {iso }}(G ; S(V))$ is closed under taking subgroups, then 
$\mathcal{H} \subset \mathcal{F}_{\text {iso }}(G ; S(V))$ and it follows that

$$
\mathcal{F}_{\text {iso }}(G ; D \backslash M)=\mathcal{F}_{\text {iso }}(G ; S(\nu)) \cup \mathcal{F}_{\text {iso }}(G ; S(V)) .
$$

If $X$ is (nonequivariantly) contractible, then so is $D$. By the construction, the boundary $\partial D$ is simply connected. Thus, by the $h$-cobordism theorem, $D$ can be chosen to be the disk of dimension $n=\operatorname{dim}\left(\tau_{M} \oplus \nu\right)+l \operatorname{dim} V \geq 6$ (cf. [P2, Remark 2.5]).

THEOREM 3.2. Let $G$ be a finite group such that $\mathcal{P}(G) \cap \mathcal{L}(G)=\emptyset$. Let $M$ be a smooth manifold and let $\nu$ be a smooth $\mathcal{L}(G)$-free $G$-vector bundle over $M$. Assume $M$ is compact, $\chi(M) \equiv 1\left(\bmod n_{G}\right)$, and $\tau_{M} \oplus \nu$ satisfies the bundle conditions (B1) and (B2). Assume also that the following two statements are true:

(1) $E$ is a finite contractible $G-C W$ complex such that $E^{G}=M$ and $\mathcal{K}$ is a family of subgroups of $G$ such that $\mathcal{K} \supset \mathcal{L}(G)$ and for each $K \in \mathcal{K}$, each connected component of $E^{K}$ either coincides with a connected component of $M$ or is disjoint from $M$.

(2) $B=\bigcup_{K \in \mathcal{K}} E^{K} \backslash M, N$ is a compact smooth $G$-manifold such that $N \supset B$ as a strong $G$-deformation retract, and when $B$ is nonempty, the tangent bundle $\tau_{N}$ of $N$ is the product bundle over $N$ whose fiber is the trivial G-module of dimension $\operatorname{dim} V_{0}^{G}$ for the fiber $V_{0}$ of $\tau_{M} \oplus \nu$ over a point $x_{0} \in M$.

Then there exists a smooth action of $G$ on a disk $D$ such that $D^{G}=M$ and $\nu_{M \subset D} \cong \nu \oplus \varepsilon_{M}^{W}$ for $W=l V(G)$ and a sufficiently large integer $l \geq 1$. In particular, the action of $G$ on $D$ is without large isotropy subgroups around $M$. The dimension of the disk $D$ equals

$$
\operatorname{dim} D=\operatorname{dim} V_{0}+l\left((|G|-1)-\sum_{G^{p}}\left(\left|G / G^{p}\right|-1\right)\right) .
$$

Moreover, $D \supset N$ as a $G$-submanifold disjoint from $M$ and $\mathcal{F}_{\text {iso }}(G ; D \backslash$ $(M \cup N))=\mathcal{M}(G)$. In addition to (1) and (2), assume that

(3) For each $P \in \mathcal{P}(G), E^{P}$ is simply connected.

Then the action of $G$ on $D$ can be constructed in such a way that for each prime $p|| G \mid$ and each $P \in \mathcal{P}_{p}(G), \pi_{1}\left(D^{P}\right)$ is a finite abelian group of order prime to $p$.

Proof. Let $Y=E \cup_{B} N$ be the sum of $E$ and $N$ along $B$. Then $Y$ has the structure of a finite contractible $G$-CW complex such that $Y^{G}=M$. Consider the quotient space $Y / M=\left(E \cup_{B} N\right) / M$ and form the wedge

$$
Y_{M}=(Y / M) \vee M
$$

of $Y / M$ and $M$ with respect to the base points $M \in Y / M$ and $x_{0} \in M$. The $G$-module $V_{0}$ is the fiber of $\tau_{M} \oplus \nu$ over $x_{0} \in M$. Set $\eta=\tau_{M} \oplus \nu$ and define 
a $G$-vector bundle $\bar{\eta}$ over $Y_{M}$ by letting $\bar{\eta}$ be $\eta$ over $M$ and the product $G$-vector bundle over $Y / M$ with fiber $V_{0}$. Since $\tau_{M} \oplus \nu$ satisfies the bundle conditions (B1) and (B2), it follows from Theorem 2.1 that there exist a finite contractible $G$-CW complex $X$ and a $G$-vector bundle $\xi$ over $X$ such that $X \supset Y_{M}$ as a $G$-invariant subcomplex,

$$
\mathcal{F}_{\text {iso }}\left(G ; X \backslash Y_{M}\right) \subset \mathcal{P}(G) \text { and }\left.\xi\right|_{Y_{M}} \cong \eta \oplus \varepsilon_{Y_{M}}^{V} \oplus \varepsilon_{Y_{M}}^{W}
$$

for a real $G$-module $V$ and $W=l V(G)$ for an integer $l \geq 1$. By Proposition 2.3, the $G$-module $V(G)$ is $\mathcal{M}(G)$-complete, and thus $\mathcal{P}(G)$-complete. According to Theorem 2.2, if $l$ is sufficiently large, there exists a $G$-subbundle $\theta$ of $\xi$ such that

$$
\theta \cong \varepsilon_{X}^{V} \text { and }\left.(\xi-\theta)\right|_{Y_{M}} \cong \eta \oplus \varepsilon_{Y_{M}}^{W}
$$

By the construction, $M \cup N \subset Y_{M} \subset X$ and $\mathcal{F}_{\text {iso }}\left(G ; Y_{M} \backslash(M \cup N)\right) \subset \mathcal{M}(G)$ because $\mathcal{M}(G)=\mathcal{S}(G) \backslash \mathcal{L}(G)$ and $\mathcal{L}(G) \subset \mathcal{K}$. Since $\mathcal{P}(G) \subset \mathcal{M}(G)$, it follows that

$$
\begin{aligned}
\mathcal{F}_{\text {iso }}(G ; X \backslash(M \cup N)) & =\mathcal{F}_{\text {iso }}\left(G ; X \backslash Y_{M}\right) \cup \mathcal{F}_{\text {iso }}\left(G ; Y_{M} \backslash(M \cup N)\right) \\
& \subset \mathcal{M}(G)=\mathcal{F}_{\text {iso }}(G ; S(V(G))) .
\end{aligned}
$$

Now, set

$$
\nu_{M}=\nu \oplus \varepsilon_{M}^{W} \text { and } \quad \nu_{N}=\varepsilon_{N}^{V_{0}-V_{0}^{G}} \oplus \varepsilon_{N}^{W} .
$$

Then $\left.(\xi-\theta)\right|_{M} \cong \tau_{M} \oplus \nu_{M}$ and $\left.(\xi-\theta)\right|_{N} \cong \tau_{N} \oplus \nu_{N}$. Theorem 3.1 applied for the pairs $\left(M \cup N, \nu_{M} \cup \nu_{N}\right)$ and $(X, \xi-\theta)$ asserts now that there exists a compact smooth $G$-manifold $D$ of the $G$-homotopy type of $X$, such that $D \supset M \cup N$ as a $G$-submanifold whose equivariant normal bundle restricts to $\nu_{M}$ over $M$ and $\nu_{N}$ over $N$ provided $l$ is sufficiently large. In particular, $\nu_{M \subset D} \cong \nu \oplus \varepsilon_{M}^{W}$ and the action of $G$ on $D$ is without large isotropy subgroups around $M \cup N$. By [LM, (2.2)],

$$
\operatorname{dim} V(G)=(|G|-1)-\sum_{G^{p}}\left(\left|G / G^{p}\right|-1\right) .
$$

Since $X$ is (nonequivariantly) contractible, $D$ can be chosen to be the disk of dimension $n=\operatorname{dim} V_{0}+l \operatorname{dim} V(G) \geq 6$. The family $\mathcal{M}(G)$ is closed under taking subgroups, and thus $\mathcal{F}_{\text {iso }}\left(G ; S\left(\nu_{M} \cup \nu_{N}\right)\right)=\mathcal{M}(G)$. Hence,

$\mathcal{F}_{\text {iso }}(G ; D \backslash(M \cup N))=\mathcal{F}_{\text {iso }}\left(G ; S\left(\nu_{M} \cup \nu_{N}\right)\right) \cup \mathcal{F}_{\text {iso }}(G ; S(V(G)))=\mathcal{M}(G)$.

If for each $P \in \mathcal{P}(G), E^{P}$ is simply connected, then so is $Y^{P}$ because $Y$ and $E$ have the same $G$-homotopy type. By Theorem 2.1, for each $P \in \mathcal{P}_{p}(G)$ and $p|| G \mid, \pi_{1}\left(X^{P}\right)$ is a finite abelian group of order prime to $p$, and the same is true for $\pi_{1}\left(D^{P}\right)$ because $D$ and $X$ have the same $G$-homotopy type.

In order to construct smooth $G$-actions on disks $D$ without large isotropy subgroups around $D^{G}$ (using Theorem 3.2), we need to construct finite contractible $G-\mathrm{CW}$ complexes with suitable properties. The following lemma 
is a partial result of [O4, Proposition 2.3]. Since the proof of [O4, Proposition 2.3] uses much deeper arguments than we need here, we include a more elementary proof of the lemma.

Lemma 3.3. Let $G$ be a finite group not of prime power order. Let $X$ be a finite $G-C W$ complex such that $X^{G}$ is nonempty and let $Y$ be a finite contractible $G-C W$ complex such that

$$
\chi\left(X^{H}\right)=\chi\left(Y^{H}\right)
$$

for each $H \in \mathcal{S}(G) \backslash \mathcal{P}(G)$. Then there exists a finite contractible $G-C W$ complex $E \supset X$ such that $\mathcal{F}_{\text {iso }}(G ; E \backslash X) \subset \mathcal{P}(G)$ and $E^{P}$ is simply connected for each $P \in \mathcal{P}(G)$.

Pr o of. By attaching a number of equivariant cells of isotropy types in $\mathcal{P}(G)$, we can modify $X$ so as to get $\operatorname{dim} X \geq 2$ and $\chi\left(X^{H}\right)=\chi\left(Y^{H}\right)$ for each nontrivial $H \in \mathcal{S}(G)$. By taking, if necessary, the unreduced double suspension of $Y$, we may assume that $Y^{G}$ is nonempty. Let $f: X \rightarrow Y$ be a constant $G$-map. By attaching a number of equivariant cells again of isotropy types in $\mathcal{P}(G)$ and extending $f$ over the attached cells, we can modify $X$ further so as to get $X^{P}$ simply connected. We also get in this way a modified $G$-map $f: X \rightarrow Y$ such that $f^{P}: X^{P} \rightarrow Y^{P}$ is a $\bmod$ $p$ homology equivalence for each $p|| G \mid$ and each nontrivial $P \in \mathcal{P}_{p}(G)$, and $f$ is $n$-connected for $n=\operatorname{dim} X$. Hence, the resulting $G$-CW complex $X$ is $(n-1)$-connected. According to Nakayama-Rim theory $([\mathrm{N}],[\mathrm{R}]$ and $\left[\mathrm{O} 4\right.$, Lemma A.10]), $H_{n}(X ; \mathbb{Z})$ is a projective $\mathbb{Z}[G]$-module. Since $Y$ is contractible, $H_{n}(X ; \mathbb{Z}) \cong H_{n+1}\left(Z_{f}, X ; \mathbb{Z}\right)$, where $Z_{f}$ is the mapping cyclider of $f$. By [O4, Lemma A.11], there exists a finite $G$-CW complex $T$ such that $T^{H}=p t$ for each $H \in \mathcal{S}(G) \backslash \mathcal{P}(G), T$ is $(d-1)$-connected for $d=\operatorname{dim} T \geq 2$, and $H_{d}(T ; \mathbb{Z}) \cong H_{n}(X ; \mathbb{Z})$. By taking, if necessary, the reduced suspension of $T$, we may assume that $n-d \equiv 1(\bmod 2)$. Now, by attaching a number of free equivariant cells, we can build up the wedge $X \vee T$ into a finite contractible $G$-CW complex $E$. By the construction, $\mathcal{F}_{\text {iso }}(G ; T \backslash p t) \subset \mathcal{P}(G)$, and thus $\mathcal{F}_{\text {iso }}(G ; E \backslash X) \subset \mathcal{P}(G)$.

LemMA 3.4. Let $G$ be a finite group not of prime power order. Let $F$ be a finite nonempty $C W$ complex with $\chi(F) \equiv 1\left(\bmod n_{G}\right)$. Then there exists a finite contractible $G-C W$ complex $E$ with $E^{G}=F$ such that $E^{P}$ is simply connected for each $P \in \mathcal{P}(G)$, and for each $H \in \mathcal{S}(G) \backslash \mathcal{P}(G)$, each connected component of $E^{H}$ either coincides with a connected component of $F$ or is disjoint from $F$, and then it has dimension $\leq 1$ (moreover, it can be chosen to be a wedge of circles or a point).

Proof. By the assumption $\chi(F) \equiv 1\left(\bmod n_{G}\right)$. According to [O1, Corollary on p. 167], there exists a finite contractible $G$-CW complex $Y$ such that $Y^{G}=F$. It follows that $\chi\left(Y^{G}\right)=1+k n_{G}$ for an integer $k$, and 
for each $H \in \mathcal{S}(G) \backslash\{G\}$, we have

$\chi\left(Y^{H}\right)=1+\sum_{K \geq H} \chi\left(Y^{K}, Y^{>K}\right) \quad$ and $\quad \chi\left(Y^{H}, Y^{>H}\right) \equiv 0\left(\bmod \left|N_{G}(H) / H\right|\right)$

(cf. [O1, Lemma 2]). Thus, by taking the disjoint union of $F$ and a number of equivariant 0-cells $G / H$, and attaching a number of equivariant 1-cells $G / H \times D^{1}$ to the added 0-cells for all $(H) \subset \mathcal{S}(G) \backslash\{G\}$, we can obtain a finite $G$-CW complex $X$ such that $X^{G}=F$ and $\chi\left(X^{H}\right)=\chi\left(Y^{H}\right)$ for all $H \in \mathcal{S}(G)$. In particular, each 1-cell $G / H \times D^{1}$ can be attached via a $G$-map from $G / H \times S^{0}$ that is constant on each copy $\{g H\} \times S^{0}$ of the sphere $S^{0}$. According to Lemma 3.3, there exists a finite contractible $G$-CW complex $E \supset X$ such that $\mathcal{F}_{\text {iso }}(G ; E \backslash X) \subset \mathcal{P}(G)$ and $E^{P}$ is simply connected for each $P \in \mathcal{P}(G)$. Therefore, $E^{G}=X^{G}=F$ and $E^{H}=X^{H}$ for each $H \in \mathcal{S}(G) \backslash \mathcal{P}(G)$. By the construction, for each $H \in \mathcal{S}(G) \backslash \mathcal{P}(G)$, we have $\operatorname{dim}(X \backslash F) \leq 1$ and each connected component of $E^{H}$ either coincides with a connected component of $F$ or is disjoint from $F$, and then it has dimension $\leq 1$ and can be chosen to be a wedge of circles or a point.

A finite group $G$ is called a pseudocyclic group if $G$ has a normal subgroup $P \in \mathcal{P}(G)$ such that $G / P$ is cyclic. Let $\mathcal{C}_{\mathcal{P}}$ be the class (denoted by $\mathcal{G}^{1}$ in $[\mathrm{O} 1])$ of all finite pseudocyclic groups. For a finite group $G$, let $\mathcal{C}_{\mathcal{P}}(G)$ denote the family of pseudocyclic subgroups of $G$. For a finite group $G$ not of prime power order, $n_{G}=0$ if and only if $G \in \mathcal{C}_{\mathcal{P}}$ (cf. [O1, Corollary on p. 171]). For a prime $p$ and an integer $q \geq 2$ such that $p$ does not divide $q$, the abelian group $G=\mathbb{Z}_{p q} \times \mathbb{Z}_{p}$ is a pseudocyclic group that is neither cyclic nor of prime power order.

Lemma 3.5. Let $G$ be a finite group not of prime power order, and assume $n_{G}=0$. Let $F$ be a finite nonempty $C W$ complex with $\chi(F)=1$. Then there exists a finite contractible $G-C W$ complex $E$ with $E^{G}=F$ such that $\mathcal{F}_{\text {iso }}(G ; E \backslash F) \subset \mathcal{P}(G)$ and $E^{P}$ is simply connected for each $P \in \mathcal{P}(G)$.

Proof. According to [O1, Corollary on p. 167], there exists a finite contractible $G$-CW complex $Y$ such that $Y^{G}=F$. Since $n_{G}=0$, we have $n_{H}=0$ for each $H \in \mathcal{S}(G) \backslash \mathcal{P}(G)$, and it follows that $\chi\left(Y^{H}\right)=1$ for each $H \in \mathcal{S}(G)$. Set $X=F$ and consider the trivial action of $G$ on $X$. Then $\chi\left(X^{H}\right)=\chi\left(Y^{H}\right)$ for each $H \in \mathcal{S}(G)$, and Lemma 3.3 yields a finite contractible $G$-CW complex $E$ with $E^{G}=F$ such that $\mathcal{F}_{\text {iso }}(G ; E \backslash F) \subset$ $\mathcal{P}(G)$ and $E^{P}$ is simply connected for each $P \in \mathcal{P}(G)$.

Lemma 3.6. Let $G$ be a finite group not of prime power order, and assume $n_{G}>0$. Let $F$ be a finite nonempty $C W$ complex with $\chi(F) \equiv 1$ $\left(\bmod n_{G}\right)$. Then there exists a finite contractible $G-C W$ complex $E$ with $E^{G}=F$ such that $E^{P}$ is simply connected for each $P \in \mathcal{P}(G)$, and for each 
$H \in \mathcal{S}(G) \backslash \mathcal{C}_{\mathcal{P}}(G)$, each connected component of $E^{H}$ either coincides with a connected component of $F$ or is a point outside $F$.

Pr o of. Set $\mathcal{B}(G)=\mathcal{S}(G) \backslash\left(\mathcal{C}_{\mathcal{P}}(G) \cup\{G\}\right)$. According to [O1, Corollary on p. 167], there exists a finite contractible $G$-CW complex $Y_{G}$ such that $Y_{G} \supset F$ as the $G$-fixed point set and for each $(H) \subset \mathcal{B}(G)$, there exists a finite contractible $H$-CW complex $Y_{(H)}$ whose $H$-fixed point set consists of exactly $n_{H}+1$ points. Since $H$ is not a pseudocyclic group, $n_{H}>0$. Hence, the $H$-fixed point set of $Y_{(H)}$ consists of two or more isolated points. Choose a base $G$-fixed point $y_{G}$ in $Y_{G}$ and for each $(H) \subset \mathcal{B}(G)$, choose a base $H$-fixed point $y_{(H)}$ in $Y_{(H)}$ and an integer $b_{(H)}>0$. Now, form the wedge

$$
Y=Y_{G} \vee \bigvee_{(H) \subset \mathcal{B}(G)}\left(\bigvee_{i=1}^{b_{(H)}}\left(G \times_{H} Y_{(H)}\right) /\left(G \times_{H}\left\{y_{(H)}\right\}\right)\right) .
$$

Then $Y$ is a finite contractible $G$-CW complex such that $Y^{G}=F$, and it follows that $\chi\left(Y^{G}\right)=1+k n_{G}$ for an integer $k$. Moreover, for each $H \in$ $\mathcal{S}(G) \backslash\{G\}$,

$\chi\left(Y^{H}\right)=1+\sum_{K \geq H} \chi\left(Y^{K}, Y^{>K}\right) \quad$ and $\quad \chi\left(Y^{H}, Y^{>H}\right) \equiv 0\left(\bmod \left|N_{G}(H) / H\right|\right)$

(cf. [O1, Lemma 2]). Choose the integers $b_{(H)}$ so that $\chi\left(Y^{H}, Y^{>H}\right)>0$ for $(H) \subset \mathcal{B}(G)$. For each $(H) \subset \mathcal{S}(G)$, set $c_{(H)}=\chi\left(Y^{H}, Y^{>H}\right) /\left|N_{G}(H) / H\right|$ and

$$
j_{(H)}= \begin{cases}0 & \text { for } c_{(H)} \geq 0 \\ 1 & \text { for } c_{(H)}<0\end{cases}
$$

Note that $c_{(H)}>0$ and thus $j_{(H)}=0$ for each $(H) \subset \mathcal{S}(G) \backslash \mathcal{C}_{\mathcal{P}}(G)$. For any $H \in \mathcal{S}(G)$, set $\Sigma^{0}(G / H)^{+}=(G / H)^{+}=G / H \amalg p t$ and write $\Sigma^{1}(G / H)^{+}$ for the reduced suspension of $(G / H)^{+}$. Form the wedge

$$
X=F \vee \bigvee_{(H) \subset \mathcal{S}(G), c_{(H)} \neq 0}\left(\bigvee_{i=1}^{\left|c_{(H)}\right|} \Sigma^{j_{(H)}}(G / H)^{+}\right) .
$$

Then $X$ is a finite $G$-CW complex such that $X^{G}=F$ and $\chi\left(X^{H}\right)=\chi\left(Y^{H}\right)$ for each $H \in \mathcal{S}(G)$. According to Lemma 3.3, there exists a finite contractible $G$-CW complex $E \supset X$ such that $\mathcal{F}_{\text {iso }}(G ; E \backslash X) \subset \mathcal{P}(G)$ and $E^{P}$ is simply connected for each $P \in \mathcal{P}(G)$. By the construction, $E^{G}=X^{G}=F$ and $E^{H}=X^{H}$ for each $H \in \mathcal{S}(G) \backslash \mathcal{P}(G)$. Moreover, for each $(H) \subset \mathcal{B}(G)$, $E^{=H}$ is the disjoint union of $c_{(H)}$ copies of $(G / H)=H$. Hence, for each $H \in$ $\mathcal{S}(G) \backslash \mathcal{C}_{\mathcal{P}}(G)$, each connected component of $E^{H}$ either coincides with a connected component of $F$ or is a point outside $F$.

Let $G$ be a finite Oliver group (that is, $n_{G}=1$ ). Then $\mathcal{P}(G) \subset \mathcal{C}_{\mathcal{P}}(G) \subset$ $\mathcal{M}(G)$ (cf. [LM, Proposition 1.2]), and thus $\mathcal{C}_{\mathcal{P}}(G) \cap \mathcal{L}(G)=\emptyset$; that is, no 
large subgroup of $G$ is pseudocyclic. Hence, if in Lemma 3.6 we assume that $G$ is a finite Oliver group, then there is no Euler characteristic restriction on $E^{G}=F$ and for each $L \in \mathcal{L}(G)$, each connected component of $E^{L}$ either coincides with a connected component of $F$ or is a point outside $F$. It turns out that if $G$ is a finite Dress-Oliver group, we can construct $E$ in such a way that $E^{L}=F$ for each $L \in \mathcal{L}(G)$.

Lemma 3.7. Let $G$ be a finite Dress-Oliver group. Let $F$ be a finite nonempty $C W$ complex. Then there exists a finite contractible $G-C W$ complex $E$ such that $E^{L}=F$ for each $L \in \mathcal{L}(G)$, and $E^{H}$ is simply connected for each $H \in \mathcal{M}(G)$.

Proof. Consider all different Dress subgroups $G^{p_{1}}, \ldots, G^{p_{k}}$ of $G$. By the assumption, $n_{G^{p_{i}}}=1$ for $i=1, \ldots, k$, and by [O1], there exists a finite contractible $G^{p_{i}}$ CW complex $Y_{i}$ such that $Y_{i}^{G^{p_{i}}}=\emptyset$. Consider the induced $G$-space $X_{i}=\operatorname{Ind}_{G^{p_{i}}}^{G}\left(Y_{i}\right)$ of all $G^{p_{i}}$-maps $G \rightarrow Y_{i}$ with the action of $G$ given by $(g \varphi)\left(g^{\prime}\right)=\varphi\left(g^{\prime} g^{-1}\right)$ for any $G^{p_{i}}$-map $\varphi: G \rightarrow Y_{i}$ and all $g, g^{\prime} \in G$. Then $X_{i}$ has the structure of a finite contractible $G$-CW complex with $X_{i}^{G^{p_{i}}}=\emptyset$. Consider the cartesian product $X=X_{1} \times \ldots \times X_{k}$ with the diagonal action of $G$. Then $X$ is a finite contractible $G$-CW complex with $X^{L}=\emptyset$ for each $L \in \mathcal{L}(G)$. Now, consider the join $E=F * X * S(V(G))$ with the join action of $G$. Then $E^{L}=F$ for each $L \in \mathcal{L}(G)$. Moreover, for each $H \in \mathcal{M}(G)$, $\operatorname{dim} S(V(G))^{H} \geq 1$ by Proposition 1.9, and since $E^{H}=F * X^{H} * S(V(G))^{H}$, $E^{H}$ is simply connected.

Proof of Theorems 0.2 and 0.3. As noted in the Introduction, in Theorems 0.2 and $0.3,(1)$ implies (2) by Theorem 0.1 . Now, we show the converse implication. Let $G$ be a finite group such that $\mathcal{P}(G) \cap \mathcal{L}(G)=\emptyset$. Let $M$ be a smooth manifold and let $\nu$ be a smooth $\mathcal{L}(G)$-free $G$-vector bundle over $M$. Assume $M$ is compact, $\chi(M) \equiv 1\left(\bmod n_{G}\right)$, and $\tau_{M} \oplus \nu$ satisfies the bundle conditions (B1) and (B2).

The case $n_{G}=0$ ( $G$ is a pseudocyclic group $)$. In this case $\chi(M)=1$. Assume $M$ has a connected component of dimension $>1$ or $M$ is the disjoint union of the disk $D^{1}$ and a number $k \geq 0$ of copies of the circle $S^{1}$. Since $M$ has the structure of a finite $\mathrm{CW}$ complex, Lemma 3.5 yields a finite contractible $G$-CW complex $E$ such that $E^{G}=M$ and $\mathcal{F}_{\text {iso }}(G ; E \backslash M) \subset$ $\mathcal{P}(G)$. Set

$$
\mathcal{K}=\mathcal{S}(G) \backslash \mathcal{P}(G) \quad \text { and } \quad B=\bigcup_{H \in \mathcal{K}} E^{H} \backslash M .
$$

Then $\mathcal{K} \supset \mathcal{L}(G)$ and $B=\emptyset$. Set $N=\emptyset$ and note that the statements (1) and (2) in Theorem 3.2 are true. According to Theorem 3.2, there exists a smooth action of $G$ on a disk $D$ such that $D^{G}=M$ and $\nu_{M \subset D} \cong$ $\nu \oplus \varepsilon_{M}^{W}$ for $W=l V(G)$ and a sufficiently large integer $l \geq 1$. Moreover, 
$\mathcal{F}_{\text {iso }}(G ; D \backslash M)=\mathcal{M}(G)$. Clearly, in the case where $M=D^{n}$ for $n \geq 0$, one may set $D=D(\nu)$ to get the proof with $\mathcal{F}_{\text {iso }}(G ; D \backslash M) \subset \mathcal{M}(G)$, and set $D=D\left(\nu \oplus \varepsilon_{M}^{W}\right)$ with $W=V(G)$ to get $\mathcal{F}_{\text {iso }}(G ; D \backslash M)=\mathcal{M}(G)$.

To complete the argument for $n_{G}=0$, it remains to consider the case where $M$ is the disjoint union of a point and a number $k>0$ of copies of $S^{1}$. The bundle conditions (B1) and (B2) then imply that $G$ has two real $G$-modules $V$ and $W$ such that $\operatorname{dim} V^{G}=1, \operatorname{dim} W^{G}=0$, and $\operatorname{Res}_{P}^{G}(V) \cong$ $\operatorname{Res}_{P}^{G}(W)$ for each $P \in \mathcal{P}(G)$. By [O4], such $G$-modules $V$ and $W$ exist if and only if $G$ has a subquotient isomorphic to the dihedral group of order $2 p q$ for two coprime integers $p, q \geq 2$. Since $G$ is pseudocyclic, $G$ does not have such a subquotient, and thus this case is impossible.

The case $n_{G}=1$ ( $G$ is an Oliver group). In this case, there is no restriction on $\chi(M)$. Moreover, $\mathcal{P}(G) \subset \mathcal{C}_{\mathcal{P}}(G) \subset \mathcal{M}(G)$, and hence $\mathcal{C}_{\mathcal{P}}(G) \cap$ $\mathcal{L}(G)=\emptyset$. Now, Lemma 3.6 yields a finite contractible $G$-CW complex $E$ such that $E^{G}=M$ and for each $H \in \mathcal{S}(G) \backslash \mathcal{C}_{\mathcal{P}}(G)$, each connected component of $E^{H}$ either coincides with a connected component of $M$ or is a point outside $M$. Set

$$
\mathcal{K}=\mathcal{S}(G) \backslash \mathcal{C}_{\mathcal{P}}(G) \quad \text { and } \quad B=\bigcup_{H \in \mathcal{K}} E^{H} \backslash M .
$$

Then $\mathcal{K} \supset \mathcal{L}(G)$ and $B$ is a discrete space. Let $V_{0}$ be the fiber of $\tau_{M} \oplus \nu$ over a base point $x_{0} \in M$. For $n=\operatorname{dim} V_{0}^{G}$ and $b \in B$, take a copy $D_{b}^{n}$ of the disk $D^{n}$ and identify $b$ with the origin of $D_{b}^{n}$. Let $N$ be the disjoint union of the disks $D_{b}^{n}$ taken for all $b \in B$. Then $N$ admits the structure of a smooth $G$-manifold and the statements (1) and (2) in Theorem 3.2 are true. According to Theorem 3.2, there exists a smooth action of $G$ on a disk $D$ such that $D^{G}=M, \nu_{M \subset D} \cong \nu \oplus \varepsilon_{M}^{W}$ for $W=l V(G)$ and a sufficiently large integer $l \geq 1$, and $\mathcal{F}_{\text {iso }}(G ; D \backslash(M \cup N))=\mathcal{M}(G)$.

If $G$ is a finite Dress-Oliver group, that is, $n_{L}=1$ for each $L \in \mathcal{L}(G)$, then Lemma 3.7 yields a finite contractible $G$-CW complex $E$ such that $E^{L}=M$ for each $L \in \mathcal{L}(G)$. Set

$$
\mathcal{K}=\mathcal{S}(G) \backslash \mathcal{M}(G) \text { and } B=\bigcup_{H \in \mathcal{K}} E^{H} \backslash M .
$$

Then $\mathcal{K}=\mathcal{L}(G)$ and $B=\emptyset$. Set $N=\emptyset$ and note that, as above, the result follows by using Theorem 3.2. In particular, $\mathcal{F}_{\text {iso }}(G ; D \backslash M)=\mathcal{M}(G)$.

The case $n_{G}>1$ ( $G$ is neither a pseudocyclic group nor an Oliver group). Since $M$ has the structure of a finite CW complex, Lemma 3.4 yields a finite contractible $G$-CW complex $E$ such that $E^{G}=M$ and for each $H \in$ $\mathcal{S}(G) \backslash \mathcal{P}(G)$, each connected component of $E^{H}$ either coincides with a connected component of $M$ or is disjoint from $M$, and then it has dimension $\leq 1$ (moreover, it can be chosen to be a wedge of circles or a point). Set 


$$
\mathcal{K}=\mathcal{S}(G) \backslash \mathcal{P}(G) \quad \text { and } \quad B=\bigcup_{H \in \mathcal{K}} E^{H} \backslash M .
$$

Then $\mathcal{K} \supset \mathcal{L}(G)$ and each connected component of $B$ has dimension $\leq 1$. Choose a base point $x_{0} \in M$ and write $V_{0}$ for the fiber of $\tau_{M} \oplus \nu$ over $x_{0}$. Then $\operatorname{dim} V_{0}^{G}=\operatorname{dim} M_{0}$, where $M_{0}$ is the connected component of $M$ containing $x_{0}$. Now, assume $M$ has a connected component of dimension $>1$, so that we can assume that $\operatorname{dim} M_{0}>1$. As a result, there exists a compact smooth $G$-manifold $N$ such that $N \supset B$ as a strong $G$-deformation retract and the tangent bundle $\tau_{N}$ is the product bundle over $N$ whose fiber is the trivial $G$-module of dimension $\operatorname{dim} V_{0}^{G}$. Since the statements (1) and (2) in Theorem 3.2 are true, once again Theorem 3.2 yields the required action of $G$ on a disk $D$.

Proof of Theorems 0.4 and 0.5. In Theorems 0.4 and 0.5, (1) implies (2) by [O1] and [EL]. In fact, for a smooth action of $G$ on a disk $D$, it follows from [O1, Corollary on p. 167] that $\chi(M) \equiv 1\left(\bmod n_{G}\right)$, and according to [EL, (3.1) and (3.2)], $D^{G}$ is stably complex when $G$ has a normal Sylow 2 -subgroup. In order to show that (2) implies (3), we argue as follows. Proposition 1.6 (resp., Proposition 1.8) asserts that given $G \in \mathcal{C}_{\mathcal{N}}$ (resp., $G \in \mathcal{D}_{\mathcal{N}}$ ) and a compact stably complex smooth manifold $M$ (resp., a compact smooth manifold $M)$, there exists a smooth $\mathcal{L}(G)$-free $G$-vector bundle $\nu$ over $M$ such that $\tau_{M} \oplus \nu$ satisfies the bundle conditions (B1) and (B2). Thus, by applying Theorem 0.2 in the proof of Theorem 0.4 and applying Theorem 0.2 (resp., Theorem 0.3) in the proof of Theorem 0.5, (2) implies (3). Clearly, (3) implies (1) in Theorems 0.4 and 0.5.

Remark 3.8. In the proof of Theorems 0.2 and 0.3 , the $G$-CW complex $E$ can always be chosen so that $E^{P}$ is simply connected for each $P \in \mathcal{P}(G)$ (see Lemmas 3.4-3.7). Hence, in Theorem 3.2, the statement (3) is true and so the construction of the action of $G$ on a disk $D$ can be done in such a way that for each $P \in \mathcal{P}_{p}(G)$ and $p|| G \mid, \pi_{1}\left(D^{P}\right)$ is a finite abelian group of order prime to $p$. As a result, we may assume that in all statements about actions of $G$ on disks $D$ in Theorems $0.2-0.5, \pi_{1}\left(D^{P}\right)$ is a finite abelian group of order prime to $p$.

Acknowledgements. Discussions with Professor Katsuo Kawakubo had encouraged the authors to perform the research presented in this article. Both authors wish to express their gratitude to Professor Katsuo Kawakubo, and their sorrow caused by his death on April 24, 1999.

\section{References}

[BM] A. Bak and M. Morimoto, Equivariant surgery with middle dimensional singular sets. I, Forum Math. 8 (1996), 267-302. 
[Br] G. E. Bredon, Introduction to Compact Transformation Groups, Pure Appl. Math. 46, Academic Press, New York, 1972.

[tD] T. tom Dieck, Transformation Groups, de Gruyter Stud. in Math. 8, Walter de Gruyter, 1987.

[EL] A. L. Edmonds and R. Lee, Fixed point sets of group actions on Euclidean space, Topology 14 (1975), 339-345.

$[\mathrm{H}]$ D. Husemoller, Fibre Bundles, 3rd ed., Grad. Texts in Math. 20, Springer, New York, 1994.

[K] K. Kawakubo, The Theory of Transformation Groups, Oxford Univ. Press, Oxford, 1991.

[LM] E. Laitinen and M. Morimoto, Finite groups with smooth one fixed point actions on spheres, Forum Math. 10 (1998), 479-520.

[LMP] E. Laitinen, M. Morimoto and K. P awałowski, Deleting-Inserting Theorem for smooth actions of finite nonsolvable groups on spheres, Comment. Math. Helv. 70 (1995), 10-38.

[M1] M. Morimoto, Positioning map, equivariant surgery obstruction, and applications, Kyoto University RIMS Kokyuroku 793 (1992), 75-93.

[M2] -, Equivariant surgery theory: Construction of equivariant normal maps, Publ. Res. Inst. Math. Sci. 31 (1995), 145-167.

[M3] -, Equivariant surgery theory: Deleting-Inserting Theorem of fixed point manifolds on spheres and disks, K-Theory 15 (1998), 13-32.

[N] T. Nakay ama, On modules of trivial cohomology over a finite group, II (Finitely generated modules), Nagoya Math. J. Ser. A 12 (1957), 171-176.

[O1] R. Oliver, Fixed point sets of group actions on finite acyclic complexes, Comment. Math. Helv. 50 (1975), 155-177.

[O2] -, Smooth compact Lie group actions on disks, Math. Z. 149 (1976), 79-96.

[O3] -, G-actions on disks and permutation representations II, ibid. 157 (1977), 237263.

[O4] - Fixed point sets and tangent bundles of actions on disks and euclidean spaces, Topology 35 (1996), 583-615.

[P1] K. Pawałowski, Fixed point sets of smooth group actions on disks and Euclidean spaces. A survey, in: Geometric and Algebraic Topology, Banach Center Publ. 18, PWN, Warszawa, 1986, 165-180.

[P2] - Fixed point sets of smooth group actions on disks and Euclidean spaces, Topology 28 (1989), 273-289; Corrections: ibid. 35 (1996), 749-750.

[P3] - Chern and Pontryagin numbers in perfect symmetries of spheres, K-Theory 13 (1998), 41-55.

[R] D. S. Rim, Modules over finite groups, Ann. of Math. 69 (1959), 700-712.

Department of Mathematical and Environmental Sciences Faculty of Environmental Science and Technology Okayama University

Tsushimanaka 2-1-1

Okayama, 700 Japan

E-mail: morimoto@math.ems.okayama-u.ac.jp
Faculty of Mathematics and Computer Science Adam Mickiewicz University Jana Matejki 48/49 60-769 Poznań, Poland E-mail: kpa@math.amu.edu.pl

Received 18 May 1998; in revised form 11 May 1999 